\title{
Mixing in nearwall regions downstream of a sonic jet in a supersonic
}

\author{
crossflow at Mach 2.7 \\ Ming-bo Sun ${ }^{1 *}$, Zhi-wei $\mathrm{Hu}^{2}$ \\ ${ }^{1}$ Science and Technology on Scramjet Laboratory, National University of Defense \\ Technology, Changsha, 410073, China \\ ${ }^{2}$ Aerodynamics and Flight Mechanics, Faculty of Engineering and the Environment, \\ University of Southampton, Southampton, SO17 1BJ, UK \\ *correspondence: sunmingbo@nudt.edu.cn
}

\begin{abstract}
The mixing status downstream of a transverse sonic jet in a supersonic crossflow at a Mach number of 2.7 was studied. Direct numerical simulations were performed to investigate the transport of a passive scalar of jet fluid for jet-to-crossflow momentum flux ratios (denoted as $J$ ) of 1.85 and 5.5 based on the previous simulation (Sun M B, Hu Z W. AIAA J, 2018, 56(3):1047-1059). Results showed that a counter-rotating vortex pair (CVP) with two branches is generated in the jet nearfield, grows and breaks into small eddies in the farfield, which enhances the local mixing. A nearwall region in the jet lee between the CVP branches is identified to have a low mass fraction of the jet fluid and this region expands as $J$ increases. Analysis of the streamlines originating from the jet orifice and the crossflow suggests that the jet fluid in the downstream nearwall region is entrained by the lateral crossflow upstream of the jet, which travels around the jet and mixes with the injectants downstream of the jet. Higher $J$ leads to a lower mass fraction of the jet fluid in the nearwall region of the jet nearfield, but produces a higher mass fraction in the nearwall region of the farfield. A three-dimensional schematic of the jet wakes is presented and explains the formation of the nearwall low mass fraction zone in the jet nearfield.
\end{abstract}


Keywords: supersonic crossflow; mixing; transverse jet; direct numerical simulation

\section{Introduction}

The residence time of air and injected fuel in the internal flowpath of scramjet engines is of the order of milliseconds, thus, efficient mixing of fuel with air is essential to achieve a high combustion efficiency. Different jet-injection configurations have been proposed to enhance the fuel mixing. Injecting fuel from a wall orifice is one of the most efficient injection schemes and it is also simple to construct. A significant body of work about this topic has been conducted, as summarized in the previous reviews ${ }^{[1]-[3]}$.

Accurate estimation and in-depth physical understanding of the mixing process of jets in a supersonic crossflow are therefore important for scramjet design. BenYakar et al. ${ }^{[4]}$ studied the evolution of large scale vortices for an ethylene transverse jet injected into a supersonic crossflow at a Mach number of 3.4 with schlieren visualization, which is difficult to reveal the inherent mixing mechanism. Other researchers had tried to quantify the fuel distribution and jet penetration experimentally. Maddalena et al. ${ }^{[5]}$ investigated helium injections into a Mach 4.0 crossflow and utilized sampling probe measurements to determine the local concentration. Gruber et al. ${ }^{[6]}$ obtained the instantaneous mixing flowfield using nitric-oxide planar laser-induced fluorescence (NO-PLIF) and analysed the effects of different injection schemes. Lin et al. ${ }^{[7]}$ used time-averaged spontaneous vibrational Raman scattering to quantify injectant concentrations, whereas Mai et al ${ }^{[8]}$ conducted NO-PLIF to examine the effects of the incident shock wave on flow structures and mixing between the airstream and the injected gas downstream. Another alternative method of acetone-PLIF was used by VanLerberghe et al. ${ }^{[9]}$ and Sun et al. ${ }^{[10]}$ to 
analyze the wall-injected fuel penetration into a supersonic crossflow. These studies noticed that it is difficult to accurately quantify the mass fraction due to the dilution of the tracer species. A nano-particle laser scattering (NPLS) technique had also been used to reveal the detailed flow structures and the evolution of the jet near-field coherent structures ${ }^{[11]}$. However, NPLS can quantify neither the species mass fraction nor the concentration, since it can only reflect density variations.

Recently a number of numerical simulations were conducted and the detailed flowfield and coherent structure behavior have been examined. Kawai et al. ${ }^{[12]}$ and Rana et al. ${ }^{[13]}$ performed large eddy simulations (LES) of sonic jets in a supersonic stream and their results demonstrated Kelvin-Helmholtz $(\mathrm{K}-\mathrm{H})$ instabilities in the interface of the jet shear layer with the main airstream. In their simulations, the jet near-field region was calculated and a passive scalar was implemented to demonstrate the mixing process. Won et al. ${ }^{[14]}$ used detached-eddy simulation (DES) to reveal vortex evolution under the jet conditions of Ben-Yakar's experiment ${ }^{[4]}$. You et al. ${ }^{[15]}$ studied a jet with relatively low jet-to-crossflow momentum flux ratio $(J=0.35)$ in a supersonic flow at $\mathrm{Ma}=2.4$ by using DES and the Reynolds-averaged Navier-Stokes (RANS) methods. $J$ is defined as $\rho_{j} U_{j}{ }^{2} / \rho_{\infty} U_{\infty}{ }^{2}$, where the subscripts ' $j$ ' and ' $\infty$ ' denote the jet exit flow and freestream parameters, respectively. Their results suggested that the RANS approaches gave a $25 \%$ over-estimation of the mixing efficiency compared to DES. Boles et al. ${ }^{[16]}$ numerically studied transverse injection of air, helium, and ethylene into a Mach 1.98 crossflow of air and found that a hybrid RANS/LES method showed reasonably good agreement with time-averaged Mie-scattering images of the plume structure for both helium and air injection and with experimental surface pressure distributions. Hassan et al. ${ }^{[17]}$ summarized the computation of transverse jet using RANS and hybrid RANS/LES in a review and concluded in 
general hybrid RANS/LES performs better. Chai et al. ${ }^{[18]}$ conducted LES of sonic jets in supersonic crossflows and found that simulations with a turbulent inflow condition agreed better with experiments. Their simulations indicated that the flow had a global oscillation frequency associated with the shedding of vortices from the leading and trailing shear layers. In our previous work ${ }^{[19][20]}$, a hybrid RANS/LES method was validated and used to study the three-dimensional jet mixing and the effects of a micro-ramp on the jet mixing in a supersonic crossflow. Recently, our numerical simulations for JISC (jet interaction in a supersonic crossflow) clarified the upper trailing counter-rotating vortex pair $(\mathrm{CVP})^{[21]}$ and the surface trailing counter-rotating vortex pair $^{[22]}$. Upper trailing $\mathrm{CVP}^{[21]}$ is located above the major CVP and found to be related to the baroclinic effects caused by the deflecting Mach disk. Surface trailing $\mathrm{CVP}^{[22]}$ is located under the major CVP and found to be related to the herringbone separation bubble induced by the jet wake collision shock waves.

One limitation of the existing numerical results is that most of the computations only concerns the jet plume. Flow structures, mixing characteristics in the nearwall region, and the mixing process of the injectants, have not been completely revealed. Recently some researchers paid attention to the nearwall mixing while limitations of the simulation were found. For example, Ferrante et al. ${ }^{[23]}$ conducted LES to study a helium sonic jet in a supersonic crossflow and showed almost no helium in the nearwall region, whereas the experiments presented in the same paper ${ }^{[23]}$ showed a non-zero mole fraction of helium below the major core of the jet plume. Accurate knowledge of the mixing is very important to understand the combustion process. For example, previous experiments ${ }^{[24]}$ observed flame propagation against the incoming supersonic flow under certain mixing condition. This flame flashback phenomenon was tested using several ethylene injection schemes ${ }^{[25]}$, which showed that the flame 
could propagate backwards at a low $J(=1.6)$, whereas at a higher $J(=2.5)$ the flame quenched immediately after the forced ignition was turned off. This revealed the sensitivity of the reaction to the injectant mixing. It was inferred that flame quenching occurred due to the higher jet penetration for larger $J(=2.5)$, which carried main part of the injectants away from the wall and caused a low injectant concentration near the wall downstream of the jet and results in an injectant lean status ${ }^{[25]}$. While this conclusion is questionable and a dispute exists nowadays whether higher $J$ would increase the downstream nearwall injectant concentration or not. To further assess this problem, detailed information on the injectant mass fraction distribution is required, which can only be revealed through accurate simulations.

In the present study, direct numerical simulation (DNS) is conducted to investigate a sonic JISC at Ma=2.7, which provides a better way to understand mixing since detailed spatial and temporal scales of the turbulence in the near-wall region can be resolved. Injectant mass fraction distribution can be well resolved as it depends highly on the mixing due to turbulent structures. This paper is organized as follows. The algorithm, simulation conditions, a description of the computational grid, the turbulent inflow generator and simulation validation are discussed in Section 2. A description of the instantaneous jet flowfield is presented in Section 3.1. Time averaged flow statistics are presented in Section 3.2 and the three dimensional (3D) streamlines are given in Section 3.3 to exhibit the injectant entrainment in the downstream nearwall zone. Section 3.4 discusses the detailed mass fraction distribution in the nearwall region quantitatively. Section 3.5 gives a 3D schematic to explain the formation of the different nearwall mixing region in the jet nearfield and farfield.

\section{Flow conditions and numerical setup}




\subsection{Numerical simulation and inflow condition}

All simulations in this paper solve the unsteady 3D compressible Navier-Stokes equations directly without any modeling, using an in-house DNS code. The code has been developed over a number of years and has been applied to studies of instability, transition and turbulence in high-speed flows (e.g. Sandham et al. ${ }^{[26][27]}$ ). Here we provide the main features of the code and explain the run conditions for the present study. Details of the governing equations of continuity, momentum, total energy for $3 \mathrm{D}$ flowfield can be find in the references ${ }^{[28][29]}$, thus are not presented here.

The air inflow parameters (shown in Table 1) are set in accordance with the $M a=2.7$ experiments of Sun et al. ${ }^{[11]}$ with a stagnation pressure $P_{0}=101,325 \mathrm{~Pa}$, and stagnation temperature $T_{0}=300 \mathrm{~K}$. The inflow bottom wall $99 \%$ boundary-layer thickness, which is the same for all simulations, is $\delta_{i}=5.12 \mathrm{~mm}$, with the compressible (including density variations) boundary-layer displacement and momentum thicknesses of $\delta_{i}{ }^{*}=1.75 \mathrm{~mm}$ and $\theta_{i}=0.38 \mathrm{~mm}$, respectively, and Reynolds numbers $R e_{\delta i^{*}}=15,367, \operatorname{Re}_{\theta \mathrm{i}}=3,337$. A sketch of the computational domain is shown in Fig.1 below.

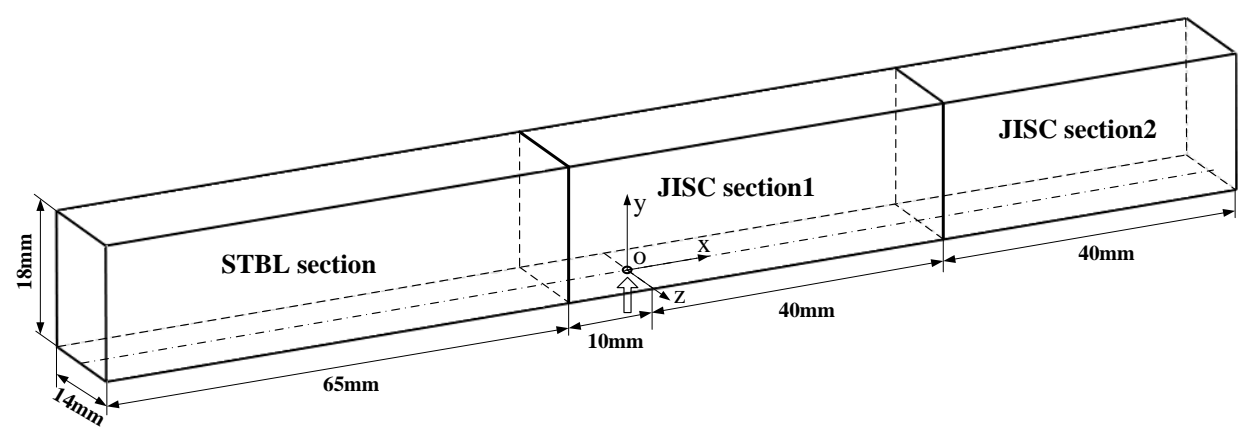

Fig.1 Schematic of the domain selected for the STBL (supersonic turbulent boundary layer) and JISC simulations. Arrow indicates the jet centre, which is also the origin of the coordinate system.

Following the numerical setup in the previous work ${ }^{[21]}$, two momentum flux 
ratios $J=1.85$ and $J=5.5$ of the air injection are modelled. Both cases lead to a sonic jet with a stagnation temperature $T_{0 \mathrm{i}}=300 \mathrm{~K}$. The jet properties are set to correspond to the injection parameters as shown in Table 1. Since the turbulence levels originating from the jet orifice are not known, a uniform profile across the jet orifice is implemented, without any added disturbances. The same setup was adopted and validated in our previous work ${ }^{[21]}$.

TABLE 1. Air jet conditions for the simulations

\begin{tabular}{lllll}
\hline Jet-to-crossflow momentum & Mach & Jet orifice & Stagnation & Stagnation \\
flux ratio & number & diameter & temperature & pressure \\
$J$ & $M a_{i}$ & $D$ & $T_{0 i}$ & $P_{0 i}$ \\
1.85 & 1.0 & $2 \mathrm{~mm}$ & $300 \mathrm{~K}$ & $110 \mathrm{kPa}$ \\
5.5 & 1.0 & $2 \mathrm{~mm}$ & $300 \mathrm{~K}$ & $330 \mathrm{kPa}$ \\
\hline
\end{tabular}

\subsection{Domain and grid distribution}

The use of a digital filter to generate the inflow boundary condition significantly reduces the length required for boundary-layer development ${ }^{[30]}$, but nevertheless there should be enough distance provided in the simulation to allow the boundary layers to adjust to an equilibrium state upstream of the jet. Wang et al. ${ }^{[31]}$ found that a distance of 12 times the inflow boundary-layer thickness is enough to obtain realistic turbulent mean and RMS profiles from the digital filter inflow generator used. Accordingly, the initial length is set to be $L_{i}=12.7 \delta_{i} \approx 65 \mathrm{~mm}$ for a fully-developed turbulence generation (the region is shown as STBL in Fig. 1, denoting the supersonic turbulent boundary layer). The jet mixing length is set to $L_{m}=90 \mathrm{~mm}$ including the nearfield (denoted as JISC section1 in Fig. 1) with $10 \mathrm{~mm}$ upstream and $40 \mathrm{~mm}$ downstream of the jet and the farfield (denoted as JISC section2) with $40 \mathrm{~mm}$ downstream of JISC section1. Therefore, the total length of the physical domain is $L_{x}=155 \mathrm{~mm}$. The wall-normal extent of the domain is $18 \mathrm{~mm}$, and a span of $14 \mathrm{~mm}$ is used. The jet is centred $75 \mathrm{~mm}$ 
downstream of the inlet with an orifice diameter of $D=2 \mathrm{~mm}$. The grid used in the current simulation is kept the same with the setup in our previous work ${ }^{[21]}$ and is briefly given as follows.

TABLE 2. Computation grids for mesh of JISC and STBL domain

\begin{tabular}{lcccllll}
\hline & $N_{x}$ & $N_{y}$ & $N_{z}$ & $\begin{array}{l}\text { Total } \\
\text { (million) }\end{array}$ & $\Delta_{x}^{+}$ & $\Delta_{y}^{+}$ & $\Delta_{z}^{+}$ \\
& & & & & & \\
\hline JISC section1 & 945 & 279 & 271 & 74.5 & $4.1-6.6$ & $0.8-10.0$ & 4.1 \\
JISC section2 & 516 & 279 & 271 & 39.0 & 6.6 & $0.8-10.0$ & 4.1 \\
STBL section & 774 & 279 & 271 & 58.5 & 6.6 & $0.8-10.0$ & 4.1 \\
\hline
\end{tabular}

Table 2 summarizes the number of grid points and resolutions in wall units, which are calculated based on the friction velocity of the incoming turbulent boundary layer at $x=-15 \mathrm{~mm}$. In wall-normal direction, the grid has $y_{1}^{+}=0.8$ for the first point off the wall and $\Delta y^{+}=5.2$ at the edge of the boundary layer and $\Delta y_{\max }^{+}=10.0$ in the mainstream. The fine mesh ensures good resolution of the near-wall turbulence. The uniform grids in the STBL and JISC2 regions have $\Delta x^{+}=6.6$, while the JISC1 region has a resolution of $\Delta x^{+}=4.1$. In the spanwise direction, grid is uniform with spacing fixed to $\Delta z^{+}=4.1$. The grid suitability for the present simulations was verified by coarsening the grid in all directions. The most sensitive feature was found to be the wall skin friction at $x=0 \mathrm{~mm}$, which varied by less than $5.6 \%$, for a grid that was coarsened by $50 \%$ in all directions. As analyzed in the previous study ${ }^{[21]}$, the grid setup ensures a fine DNS resolution in the nearwall region and a quasi-DNS (QDNS) resolution in the jet and the mainstream due to the unknown information about the turbulence in the jet orifice and also the smallest scales in the jet plume.

Large buffer layers (not shown in Fig. 1) with non-reflective outside boundaries are introduced with lengths of $15 \mathrm{~mm}, 11 \mathrm{~mm}$ and $12 \mathrm{~mm}$ at the outlet, on both the sides and the upper boundaries, respectively. The grid is smoothly stretched in the 
buffer layers towards the boundaries. The buffer layers, with coarsened mesh, help to damp fluctuations and minimize any possible reflections from the boundaries.

Non-slip and adiabatic boundary conditions are enforced on the bottom wall (shown in Fig.1). An integrated characteristic boundary condition ${ }^{[32]}$ is applied at the inflow, and an outflow condition with an integrated characteristic scheme is applied to the spanwise, top and outflow boundaries, which are located on the edge of the buffer layers. The jet orifice is defined directly in the surface wall uniform grid and set to a sonic condition with $J=1.85$ and $J=5.5$, respectively.

\subsection{Numerical methods}

The governing equations are solved using a parallel finite-difference code ${ }^{[26][27]}$. This in-house code uses a fourth-order central difference scheme for the spatial derivatives and a third-order explicit Runge-Kutta scheme for time advancement. The code makes use of an entropy splitting of the Euler terms and the Laplacian formulation of the viscous terms to improve the stability of the non-dissipative central scheme $^{[33]}$. To better resolve the steep gradient in flowfield around the injected jet, we developed a hybrid scheme which combines the entropy splitting central scheme with a central-upwind WENO-CU4 scheme and is used in the areas with high gradients ${ }^{[21]}$.

For the generation of turbulent inflow boundary conditions, Dhamankar et al. ${ }^{[34]}$ recently presented a review and broadly classified into recycling-rescaling-based methods, and synthetic turbulence generators. They evaluated several synthetic turbulence generators, comparing the required fidelity and the allowable complexity and the computational expense. They finally chose to implement a digital filter-based turbulent inflow method presented by Klein et al. ${ }^{[35]}$, using the modification of Xie and Castro $^{[30]}$ and the suggestions of Touber and Sandham ${ }^{[28][29]}$ to generate a realistic 
supersonic turbulent boundary layer at the inflow. A detailed description of the optimized digital filter used here can be found in $\operatorname{Re}^{[28]}$. The digital filter is robust to the choice of length scale, as long as the prescribed length scales are at least as large as the integral length scales of the real flow and a sufficient downstream distance is provided to allow for the turbulence to develop. In the present study, the streamwise characteristic length scales for the three velocity components $u, v$ and $w$ are set to $0.65 \delta_{\mathrm{i}}, 0.35 \delta_{\mathrm{i}}$ and $0.35 \delta_{\mathrm{i}}$ (where $\delta_{\mathrm{i}}$ denotes the $99 \%$ boundary-layer thickness at the inflow), respectively. Mean and RMS profiles are created beforehand for the wall boundary layers. The mean inflow profiles are generated from similarity solution of compressible boundary layer using the same approach as Wang et al ${ }^{[31]}$. The inflow RMS values are taken from the DNS results of Schlatter and Orlu ${ }^{[36]}$ for a similar Reynolds number.

In addition to the Navier-Stokes equations, the transport equation below for a passive scalar (mass fraction $Y_{i}$ ) of the jet fluid is solved to investigate the mixing between the jet fluid and the crossflow.

$$
\frac{\partial \rho Y_{i}}{\partial t}+\nabla \cdot\left(\rho \mathbf{u} Y_{i}\right)-\nabla\left(\rho D_{i} \nabla Y_{i}\right)=0
$$

where $\rho$ is the density, $\mathbf{u}$ is the velocity vector. $D_{i}$ is the diffusion coefficient for the passive scalar, calculated by $D_{i}=\mu /\left(\rho S_{c}\right) . S_{c}$ is the Schmidt number. The viscosity is given by Sutherland's law,

$$
\frac{\mu}{\mu_{0}}=\frac{T^{3 / 2}}{T_{s}+T}
$$

where $\mu_{0}$ is the reference viscosity for air at $T_{0}=122.1 \mathrm{~K}$ and $T_{s}=110.4 \mathrm{~K}$.

\section{Numerical results and analysis}


All simulations were performed on Archer, UK National Supercomputer. Each simulation requires approximately 180,000 core hours to run. Statistical data are based on averaging flowfields over 100 non-dimensional time units $\left(D / U_{\infty}\right)$ after running the simulations for 200 non-dimensional units to let the flow develop.

Turbulent boundary-layer mean profiles and distributions of RMS values at $x / D=-7.5$ (corresponding to the position at $60 \mathrm{~mm}$ downstream of the inlet) have been evaluated, giving a good agreement with the existing data ${ }^{[21][22]}$, validating the approach and the current calculation. Jet penetration and trajectory were also calculated and found to be reasonably consistent with the experimental correlations ${ }^{[22]}$.

\subsection{Instantaneous flow structures and mixing characteristics}

Typical contours of the instantaneous density and the jet fluid at the mid-span plane $z / D=0.0$ of $J=1.85$ and $J=5.5$ are shown in Fig. 2 . The results reveal detailed structures of the unsteady jet penetration in a $M a=2.7$ supersonic crossflow. In particular, the bow shock, barrel shock and separation bubbles are clearly identified, as have been noticed in the previous simulations ${ }^{[12]-[16]}$. It is seen that the windward structures tilt and fold as they are convected downstream. These structures engulf large amounts of free-stream fluid, and the mixing of the injectants with the freestream fluids is achieved at the periphery of the jet. Further downstream, these vortical structures break down, enhancing the local mixing process. It can be seen from Fig. 2 that there is a low injectant mass fraction region in the jet lee as the injectant is mostly moved upwards due to its wall-normal high momentum, and the size of this region increases as $J$ increases. Further downstream, the jet fluid approaches the wall and the injectant mass fraction increases compared to that in the nearfield of the jet lee. As the jet plume is fully three dimensional, it is not enough to 
conclude the mechanism of the nearwall mixing only based on the mid-span plane contours.
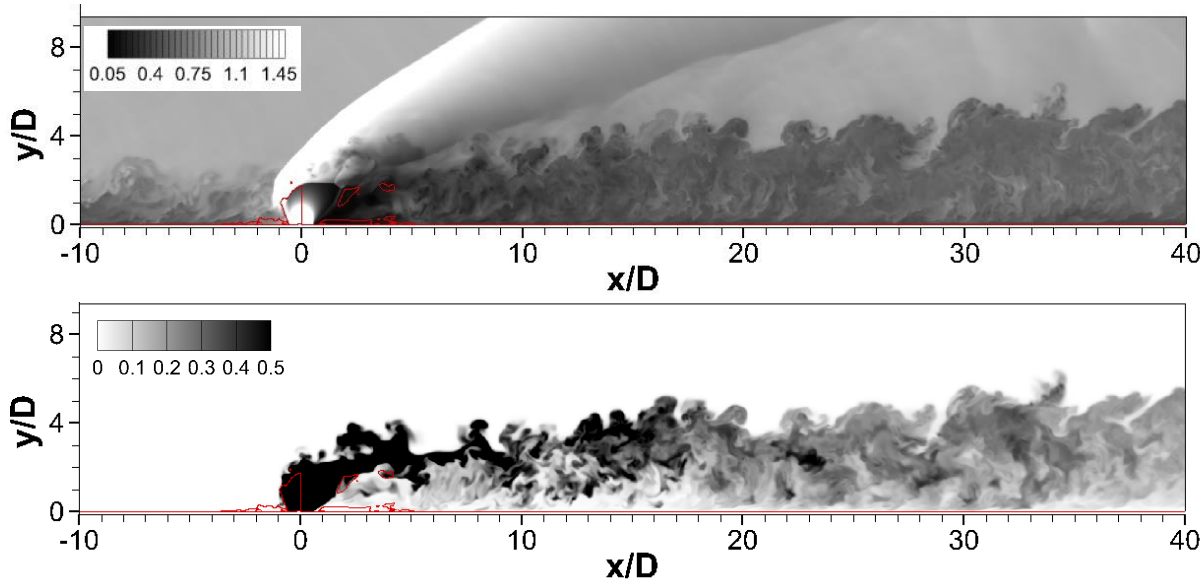

(a) $J=1.85$, density contour (top) and injectant mass fraction contour (bottom)
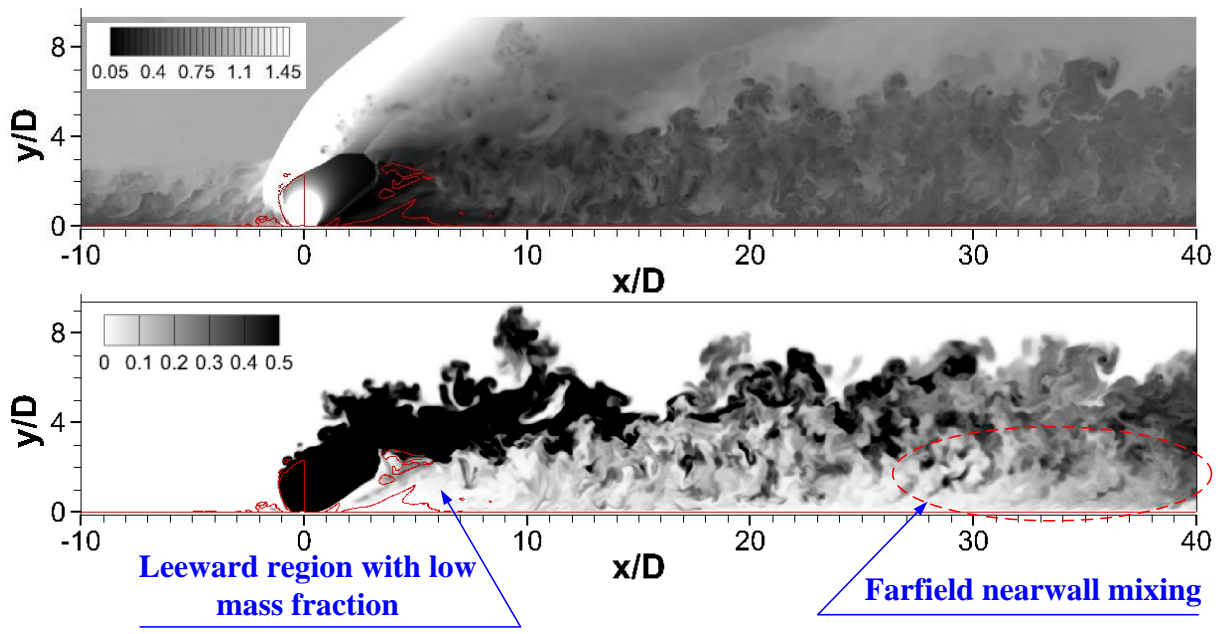

(b) $J=5.5$, density contour (top) and injectant mass fraction contour (bottom)

Fig. 2 Contours for $J=1.85$ and $J=5.5$ at the mid-span plane $(z / D=0)$. Density is normalized by the freestream density and the injectant mass fraction is normalized by the injectant mass fraction in the jet orifice. Isolines of $u / U_{\infty}=0.0$ are superposed in red.

Instantaneous injectant mass fraction contours on the cross-view planes are shown in Fig. 3 for both $J=1.85$ and $J=5.5$ cases. It is observed that downstream of the injection, there exists a region in the jet lee where the injectant mass fraction has a 
low magnitude. The jet fluid in the jet plume twists into two branches according to the effects of the major CVP and mixes with the free-stream in the nearfield. Near the wall in the jet nearfield, the jet fluid exhibits strong variation across span. In the nearwall region on the cross-plane such as $x / D=20.0$ for $J=5.5$ case, the mass fraction in the vicinity of the mid-span plane $z / D=0.0$ is lower than the regions away from $z / D=0.0$. It is clear that there exists a 'gap' between the CVP branches in the jet plume, which corresponds to the two counter rotating vortices of the major CVP. In the farfield, such as on the cross-plane $x / D=40.0$ for $J=5.5$ case, the jet fluid mixes with the air stream sufficiently and the 'gap' is smeared in the nearwall region. At $x / D=40.0$ the nearwall injectant mass fraction variation is not apparent along the span, which means the mixing in the farfield is enhanced in the nearwall region. Traditionally, it is believed that the injectant distribution is mainly dominated by the flow in the jet nearfield. These instantaneous contours are qualitative. Further discussion will be carried out with the time averaged results in the next section. 


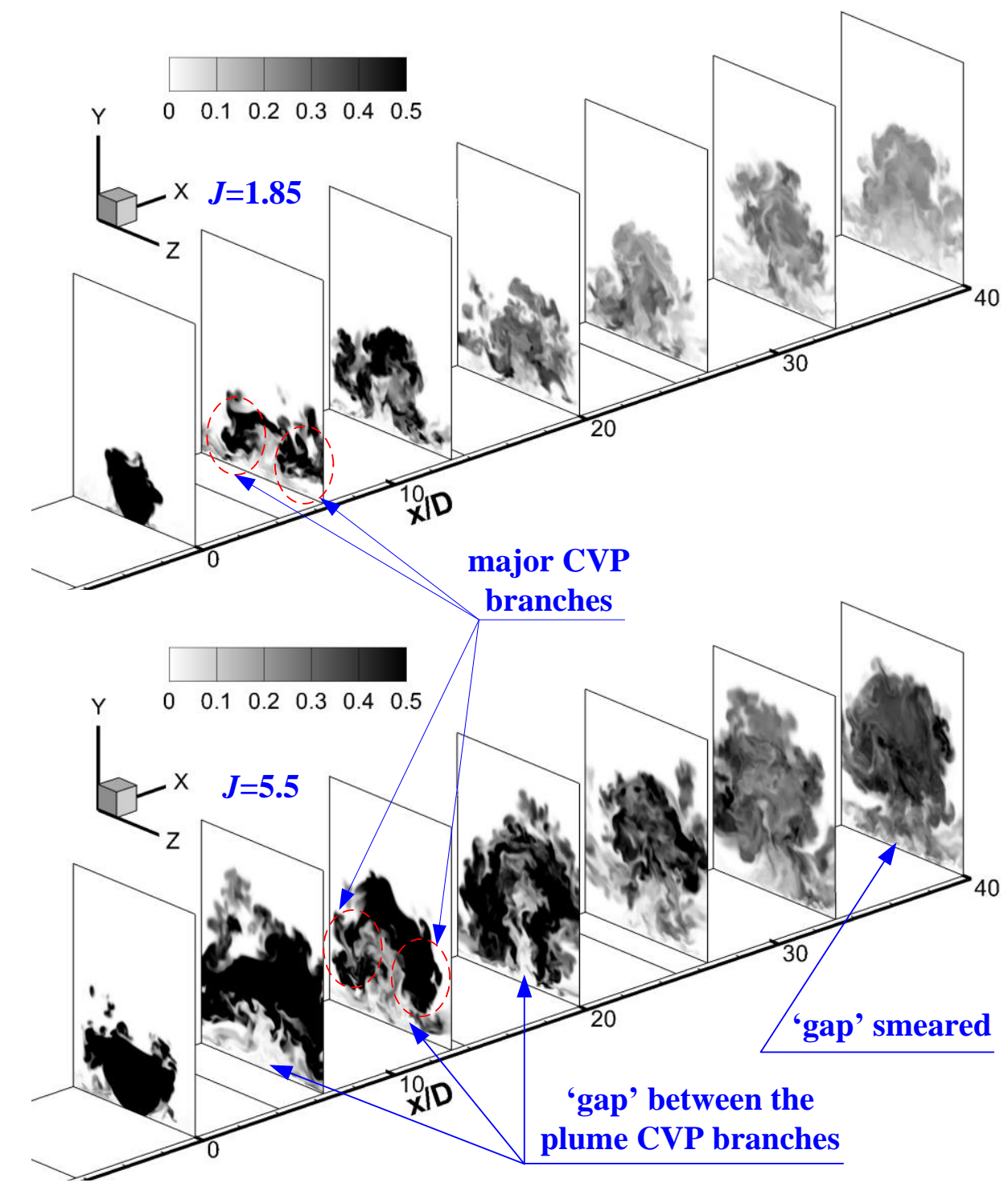

Fig.3 Instantaneous contours of injectant mass fraction of $J=1.85$ and $J=5.5$, showing cross-view planes at $x / D=0.0,6.67,13.33,20.0,26.66,33.33,40.0$

Near-wall density and injectant mass fraction contours are shown in Fig.4 on the horizontal planes. As can be seen in the slices of $y / D=0.1$ and 1.0 in Fig. 4 a)-d), the injectant is mostly distributed in the plume CVP branches of the jet for both cases. Turbulent streaks are observed in Fig.4 (a)-(b) at slices of $y / D=0.1$. There appear herringbone separation zones (wrapped by solid lines representing zero streamwise velocity) which extend both in the streamwise and spanwise direction. In the lateral downstream of the jet orifice, local density decreases significantly, which implies that expansion occurs near the jet lee. It can be seen from Fig.4(a)-(b) that the herringbone 
separation zone is confined and flow reattaches downstream of the cross point of the herringbone wings. In the vicinity of the symmetric plane, a recovery region is embayed by the herringbone separation zone near the centerline. Although similar in the flow pattern, the herringbone separation region for $J=5.5$ has a larger size than the $J=1.85$ case. It can be seen from the contours of the mass fraction that the injectant transport in the jet nearfield is affected by the nearwall separation and the recovery process directly. Little injectant is entrained into the jet leeward nearwall separation region. As the flow develops further downstream, the large pockets containing the injectant break up and significantly mix with the main airstream. In the slice $y / D=0.1$ shown in Fig.4(a), it is seen that jet fluid is entrained into the farfield zone with streaky patterns downstream of $x / D \approx 18.0$ for $J=1.85$. While the same phenomenon only occurs at $x / D \approx 30.0$ for $J=5.5$, as demonstrated by the blue dotted ellipse on the plane $y / D=0.1$ of Fig.4(b). It is inferred that the reattachment in the nearwall region of jet fluid occurs earlier for $J=1.85$ than $J=5.5$. From Fig.4(c)-(d), it is observed that the jet plume branches extend a longer streamwise distance before a fully breakdown for $J=5.5$ than $J=1.85$ at the slice of $y / D=1.0$. It is suggested that the mixing of the jet plume CVP branches with the air stream occurs more quickly for $J=1.85$ than $J=5.5$ in the nearwall region.

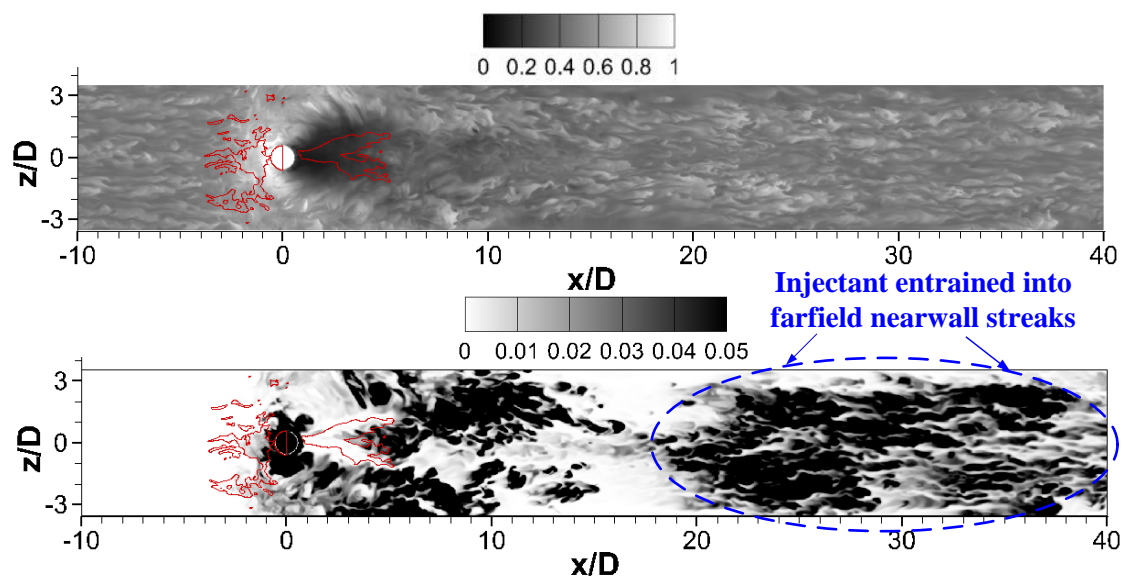

a) $y / D=0.1, J=1.85$, density(top) and injectant mass fraction (bottom) 


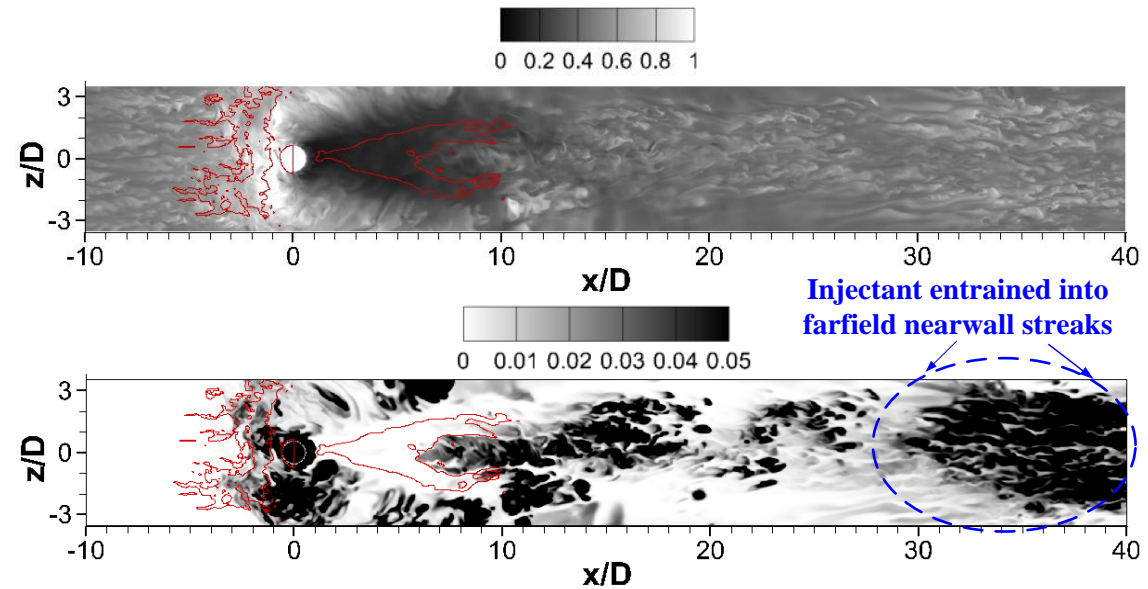

b) $y / D=0.1, J=5.5$, density(top) and injectant mass fraction (bottom)

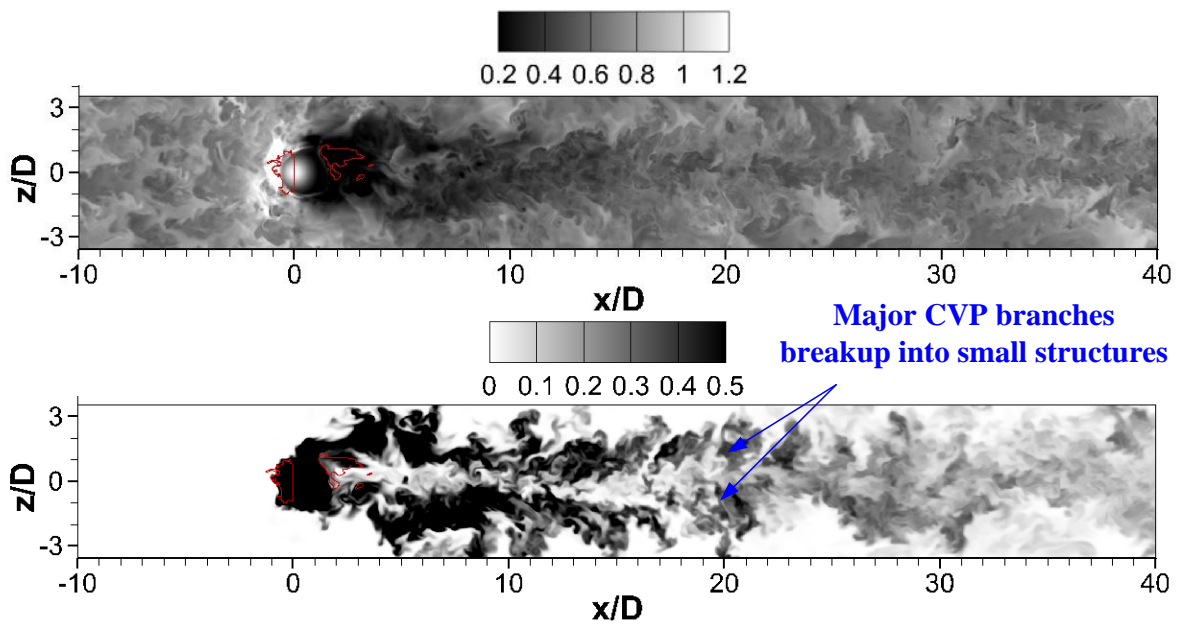

c) $y / D=1.0, J=1.85$, density (top) and injectant mass fraction (bottom)
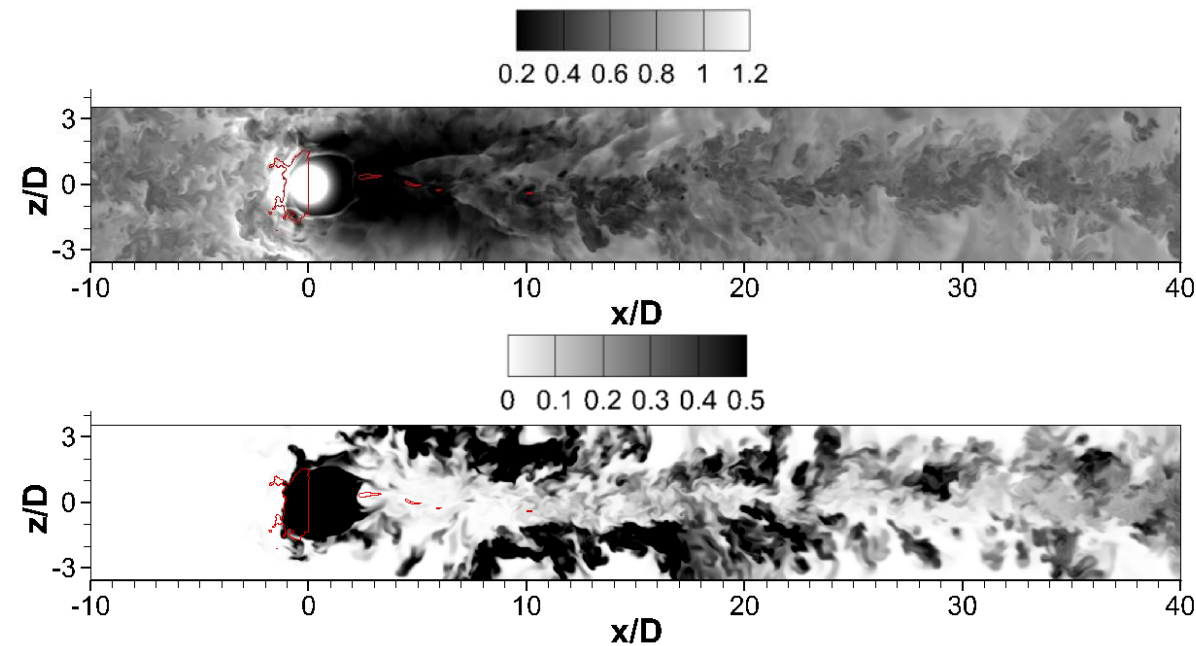

d) $y / D=1.0, J=5.5$, density (top) and injectant mass fraction (bottom) 
Fig.4 Instantaneous contours of density and injectant mass fraction of $J=1.85$ and $J=5.5$ on different wall parallel planes. Isolines of $u / U_{\infty}=0.0$ are superposed in red.

\subsection{Time-averaged results}

Time-averaged injectant mass fraction and Mach number distributions in the mid-span plane $z / D=0.0$ are shown in Fig. 5 for both cases. The jet penetrates further into the main stream as the injection momentum ratio is increased. In our previous study ${ }^{[11]}$, the jet penetration was related to the momentum ratio from experimental data,

$$
\frac{y}{D J}=\frac{2.933}{J^{0.5830}}\left(\frac{x}{D J}\right)^{0.161}
$$

where $x$ represents the streamwise distance to the jet orifice and $y$ is the nomal distance to the wall. Gruber et al. ${ }^{[37]}$ suggested a power law fit based on jet concentration identification; however Ben-yakar et al. ${ }^{[4]}$ and Sun et al. ${ }^{[11]}$ found deviation of the above power law to their experimental data. As discussed in the previous study, better agreement was achieved with the correlation of Rothstein and Wantuck $^{[38]}$,

$$
\frac{y}{D J}=J^{-0.6985} \ln \left[4.704 J\left(\frac{x}{D J}+\frac{0.6373}{J}\right)\right]
$$

Fig.5(a) shows the contour of the time-averaged injectant mass fraction for $J=1.85$ and $J=5.5$. The boundary of the penetrating jet is identified based on mass fraction $\left(Y_{\mathrm{i}}=0.02\right)$ is obtained and compared with Eqs. (3) and (4). It is seen that the current penetration line agrees well with the previous experimental correlations for both $J=1.85$ and $J=5.5$.

In Fig.5(b), in-plane streamlines are superposed on the Mach number contours. These streamlines clearly show the upstream and downstream recirculation zones of 
the jet. It can be noted from Fig.5(b) that most of the injectants passes through the windward side of the jet plume and the Mach disk and then is diverted toward the free-stream flow where the mixing occurs. A recirculation zone exists in the jet lee. It is inferred that the flow-field within the recirculation zone has a low mass exchange with the flow outside. The magnitude of the injectant mass fraction in the recirculation is smaller than outside. As seen in Fig.5(a), at $z / D=0.0$, the size of the low speed region in the jet lee becomes apparently larger as $J$ increases. The low mass fraction region wrapped by the isoline of $Y_{\mathrm{i}}=0.02$ in the nearwall region is enlarged due to the expansion of the low speed region shown in Fig.5(b). The streamlines on $z / D=0.0$ show that air freestream concentrates in the jet lee and leads to an upwash flow. The streamlines near to the leeward separation run across the low speed region and reattach to the wall. Combining with the instantaneous contours shown in Figs.34 , it is conjectured that the low mass fraction region above the jet lee separation under the jet plume is caused by the gap between the CVP branches. Further analysis of the 3D jet configuration will be carried out next.
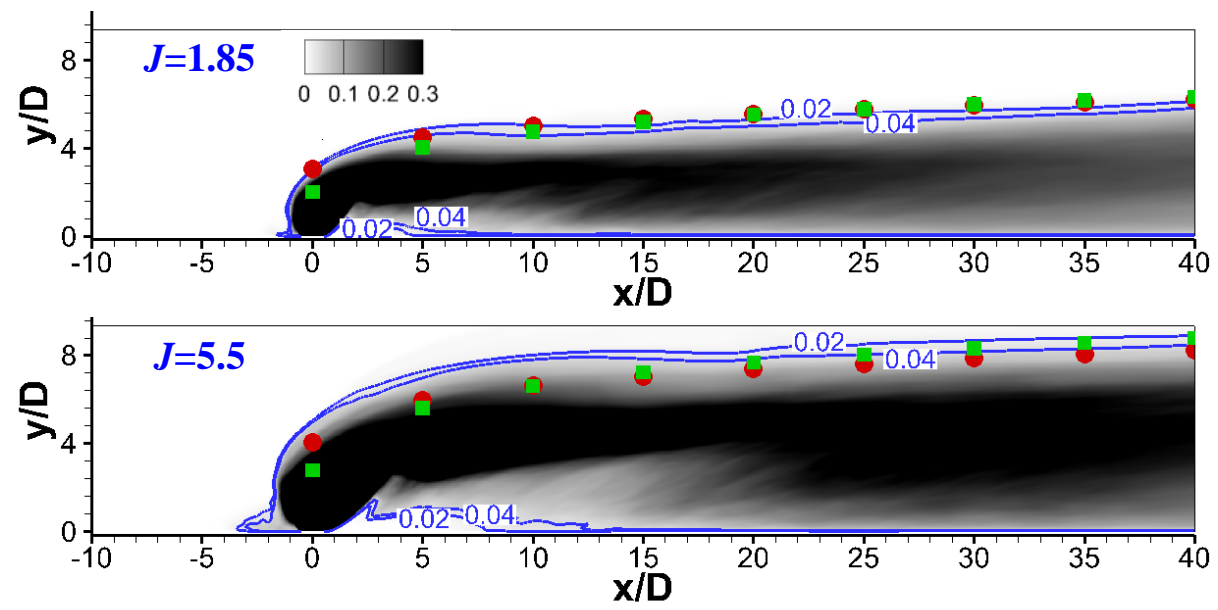

(a) Jet fluid mass fraction contours and iso-lines for $J=1.85$ and $J=5.5$ cases, $z / D=0.0$,

represents data from penetration correlation (3), $\square$ represents data from penetration correlation (4) 

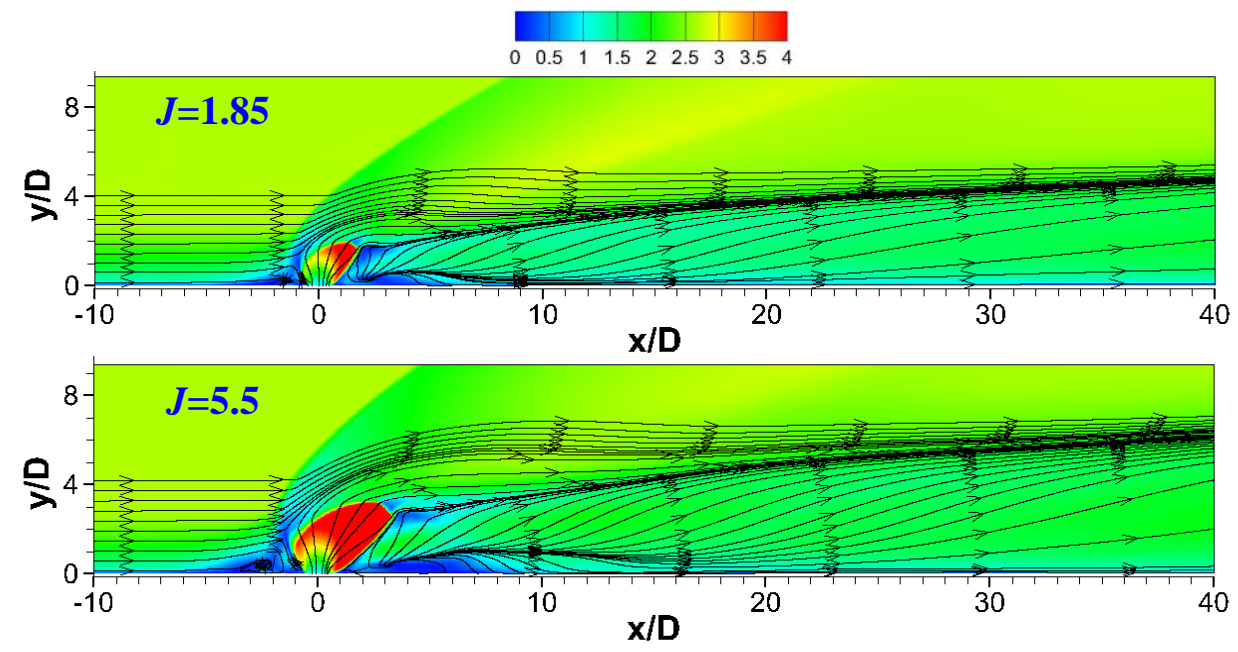

(b) Mach number contours for $J=1.85$ and $J=5.5$ cases, $z / D=0.0$

Fig.5 Time-averaged mass fraction and Mach number distribution contours on the mid-span $z / D=0.0$ plane for $J=1.85$ and $J=5.5$.

Fig.6 shows the time-averaged injectant mass fraction on different cross-sections, which is consistent with the major CVP structure. Downstream of the jet, the region of the well-mixed injectant increases as the major CVP increases in size for larger $J$. The major CVP is smeared by the turbulence originating from the jet CVP breakdown. A careful observation shows that the main body of the jet plume penetrates further in the nearfield $(x / D<20.0)$ and the gap between the plume CVP branches is enlarged for $J=5.5$ compared to $J=1.85$. The injectant in the jet farfield distributes more uniformly than the jet nearfield. The jet fluid in the nearwall region has a lower concentration than that in the plume core. The jet flow needs to be analyzed thoroughly to clarify the mixing process and the farfield injectant distribution.

The time-averaged injectant distributions on wall-parallel planes $y / D=0.1,1.0$ and 2.0 are shown in Fig.7. A herringbone separation zone is formed in the jet leeward region. As seen from the comparison of the separation zone of $J=5.5$ with $J=1.85$ at $y / D=0.1$ shown in Fig.7(a), the jet leeward separation has an increased size as $J$ increases. As discussed in Section 3.2, in the separation zone mass exchange with 
the outer flow decreases, which leads to a larger zone of low injectant mass fraction for $J=5.5$ in the jet lee than $J=1.85$. It is clear that at $y / D=0.1$ there is little injectant entrained into the jet leeward nearwall separation. In the recovery zone, the injectant mass fraction increases obviously compared to that in the separation zone. At $y / D=1.0$, the injectant rich zone is near to the jet orifice and the injectant is diluted in the farfield. Along the centerline downstream of the jet, there exists a low injectant mass fraction zone, which corresponds to the gap between the major CVP branches, as also shown in Fig.6. For $J=5.5$, the injectant mass fraction in the jet lee is clearly smaller, and the main pockets of the jet fluid, indicated by the dotted ellipse in Fig 7(b), are located further downstream than $J=1.85$ on $y / D=1.0$. It is clear that the concentrated injectant rich region of $J=1.85$ locates in an upstream position compared to $J=5.5$. This is mainly due to the smaller penetration height of lower $J$, as shown in Fig. 5. In the farfield at $y / D=1.0$ and 2.0 , as shown in Fig.7(b)-(c), the magnitude of the injectant mass fraction of $J=5.5$ is higher than $J=1.85$. It is very interesting since this indicates that the magnitude of the injectant mass fraction changes differently in the nearfield and farfield when $J$ increases.

Contours of the time-averaged Mach number with in-plane streamlines on the wall-parallel plane $y / D=1.0$ are shown in Fig.8. On this plane the streamlines run around the jet body and merge downstream in the jet farfield. The streamlines are similar on the slices of $y / D=0.1$ and 2.0 (not shown here for brevity). Together with the results shown in Fig.7, it is concluded that the jet fluid convection corresponds to the streamlines but the local mass fraction is determined by the injectant regions penetrated by the streamlines.

Fig.6 and Fig.7 show that there is a 3D low-injectant region existing in the nearfield under the jet plume, as indicated in Fig.6 by dotted lines on different cross 
sections. This region consists of the gap between the plume CVP branches and the leeward recirculation zone near the wall. When $J$ increases, the jet fluid concentration in this region (denoted as Region I in this paper) tends to decrease, which is opposite to the trend of other regions (denoted as Region II) in the flowfield. It is inferred that the jet leeward separation zone in Region I locates near the wall and has an increased size as $J$ increases. The size increase in the jet lee separation zone would push the injectant further away from the wall and form a zone with low injectant mass fraction. The other part of Region I over the separation zone increases with $J$ since the jet penetration is enhanced and the gap between plume CVP branches is enlarged, as seen from Fig.6 for $J=5.5$ compared to $J=1.85$. The injectant mass fraction in this 'gap' is lower than that in the lateral side, as demonstrated in Fig.6 and Fig.7.

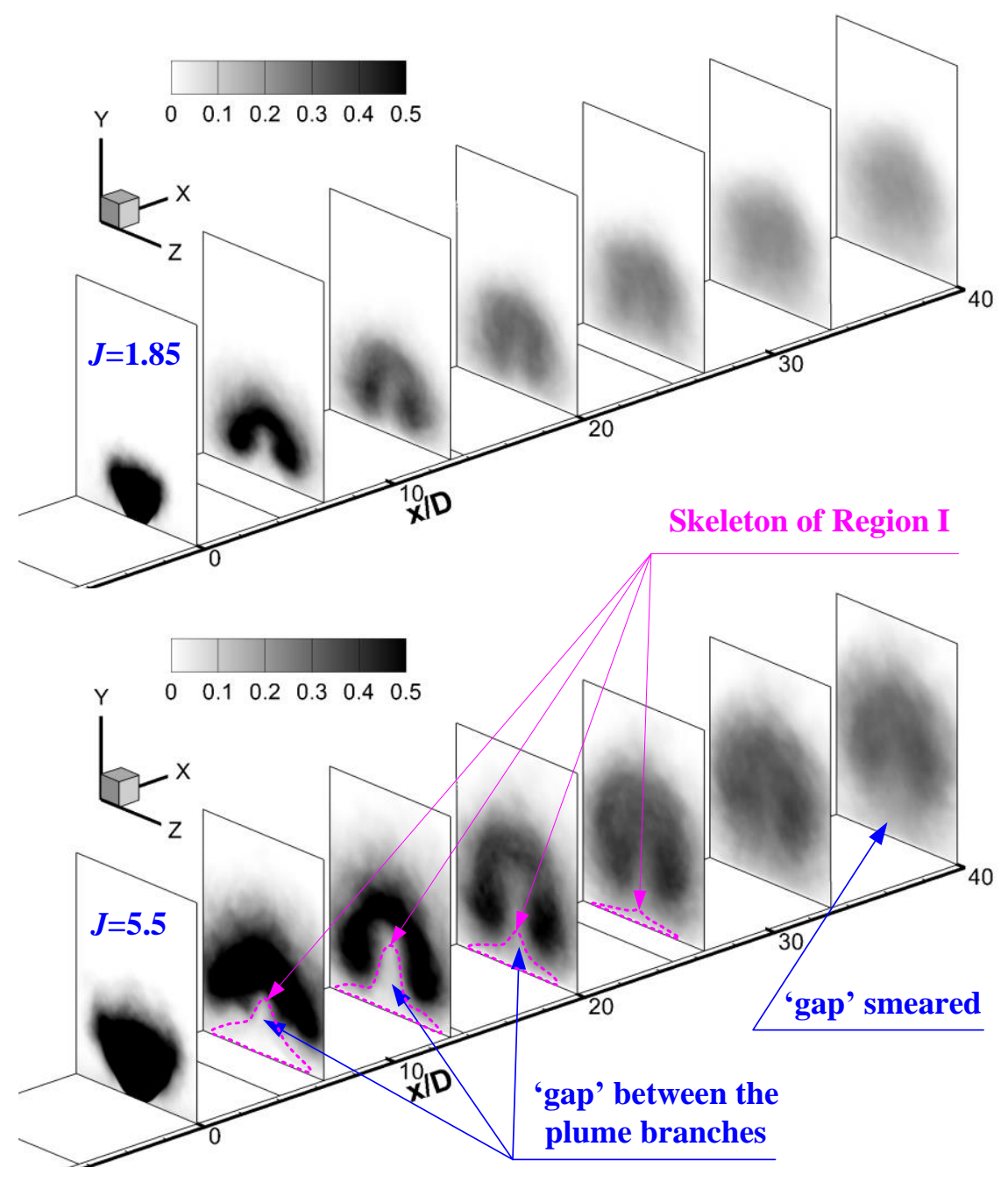


Fig.6 Time averaged contours of injectant mass fraction of $J=1.85$ and $J=5.5$, showing cross-view planes at $x / D=0.0,6.67,13.33,20.0,26.66,33.33,40.0$

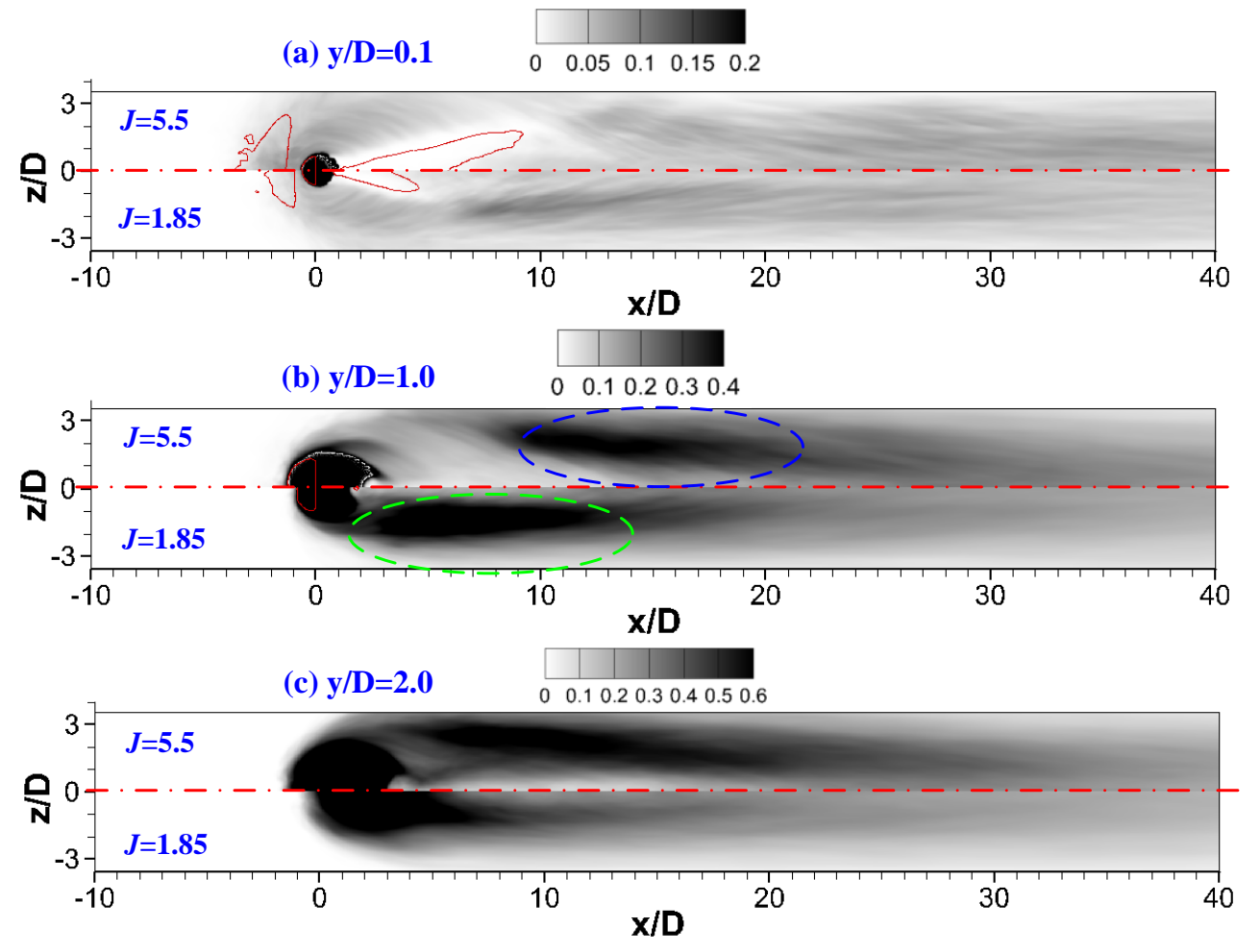

Fig.7 Comparison of the time-averaged injectant mass fraction contours on the $y / D=0.1,1.0,2.0$ planes for both $J=1.85$ and $J=5.5$

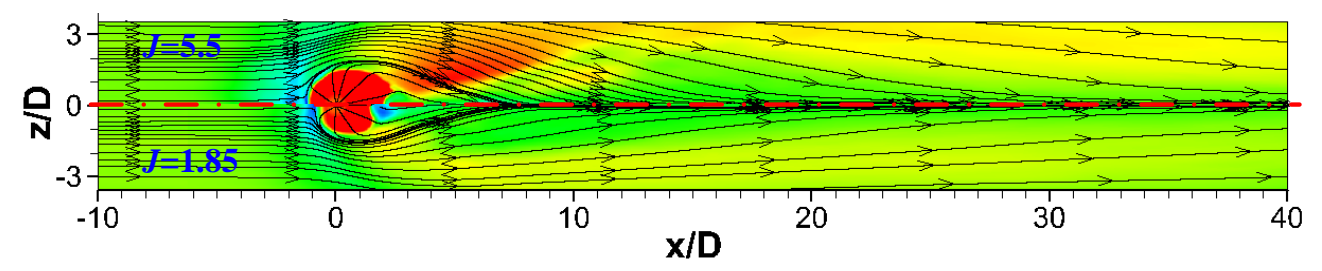

Fig.8 Comparison of the time-averaged Mach number contour with streamlines on the $y / D=1.0$ planes between $J=1.85$ and $J=5.5$

Fig.9 show the contours of the local Mach number with in-plane streamlines and injectant mass fraction in the cross sections downstream of the jet plume. At $x / D=6.0$, the major and surface trailing CVPs near the wall appear due to the interaction of the crossflow with the jet fluid. The major CVP grows in size further downstream. The 
surface trailing CVP is located in Region I (shown in the dotted ellipse in Fig.9(c)) and disappears (shown in Fig.9(d)) as the jet plume is lifted up downstream. It can be clearly seen in Fig. 9 that the major CVP increases in size for $J=5.5$ and the jet plume has a larger width and height of $J=5.5$ than $J=1.85$. At $x / D=6.0$ it is seen that the injectant mass fraction of $J=5.5$ in the plume is larger than $J=1.85$ while the injectant mass fraction in the nearwall region is lower for $J=5.5$. In the farfield $(x / D=27.5)$ of $J=5.5$ the injectant mass fraction has a higher value both in the plume and the nearwall region.

The above analysis shows that for lower $J(=1.85)$ Region I remains in a smaller size and the jet penetration height is smaller, which results in an induced nearwall zone with higher injectant mass fraction in Region I. Higher $J(=5.5)$ brings much more injectant close to the wall downstream of Region I and therefore a higher injectant mass fraction is expected. Especially in the farfield, flow runs over the jet leeward separation zone and recovers, where the effect of Region I vanishes and the local mixing is promoted by small vortices from the breakup of the major and trailing CVPs. The injectants are well mixed and have a higher mass fraction magnitude in the farfield for higher $J(=5.5)$, including the nearwall region. 

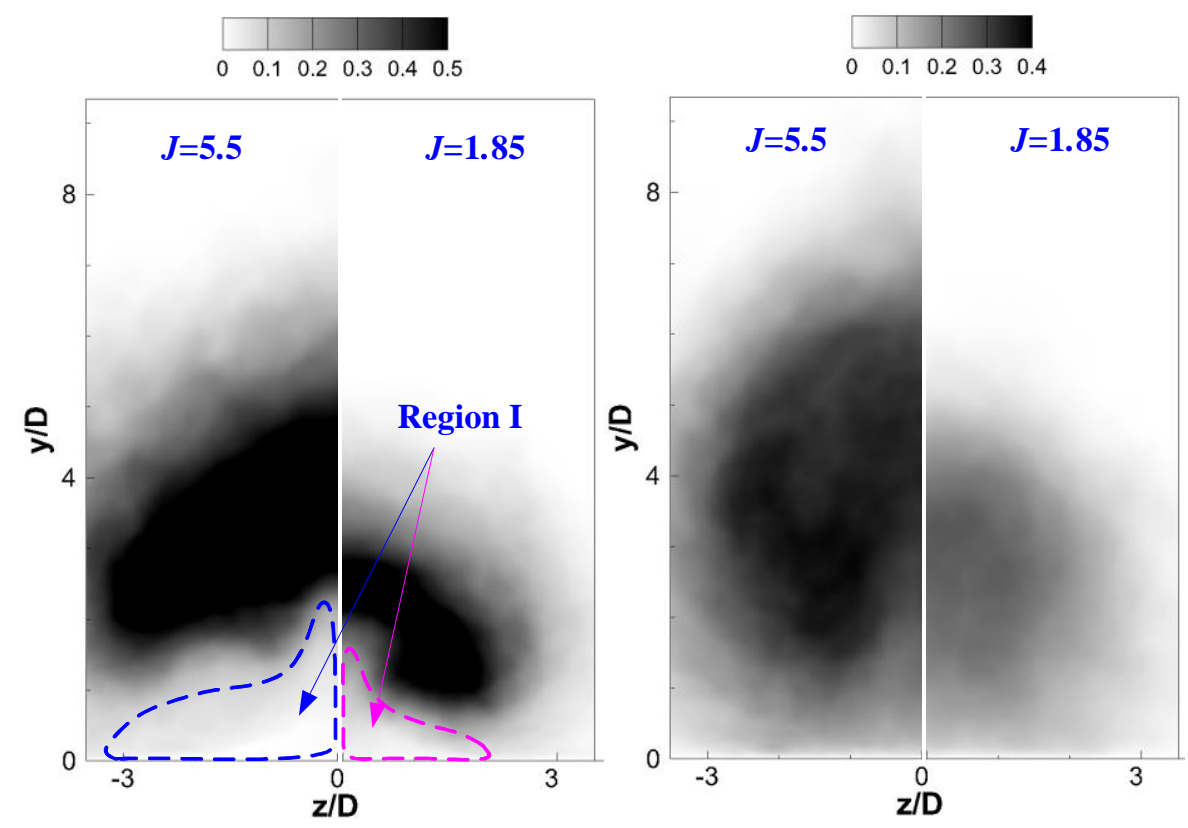

(a) $x / D=6.0$, jet fluid distribution

(b) $x / D=27.5$, jet fluid distribution
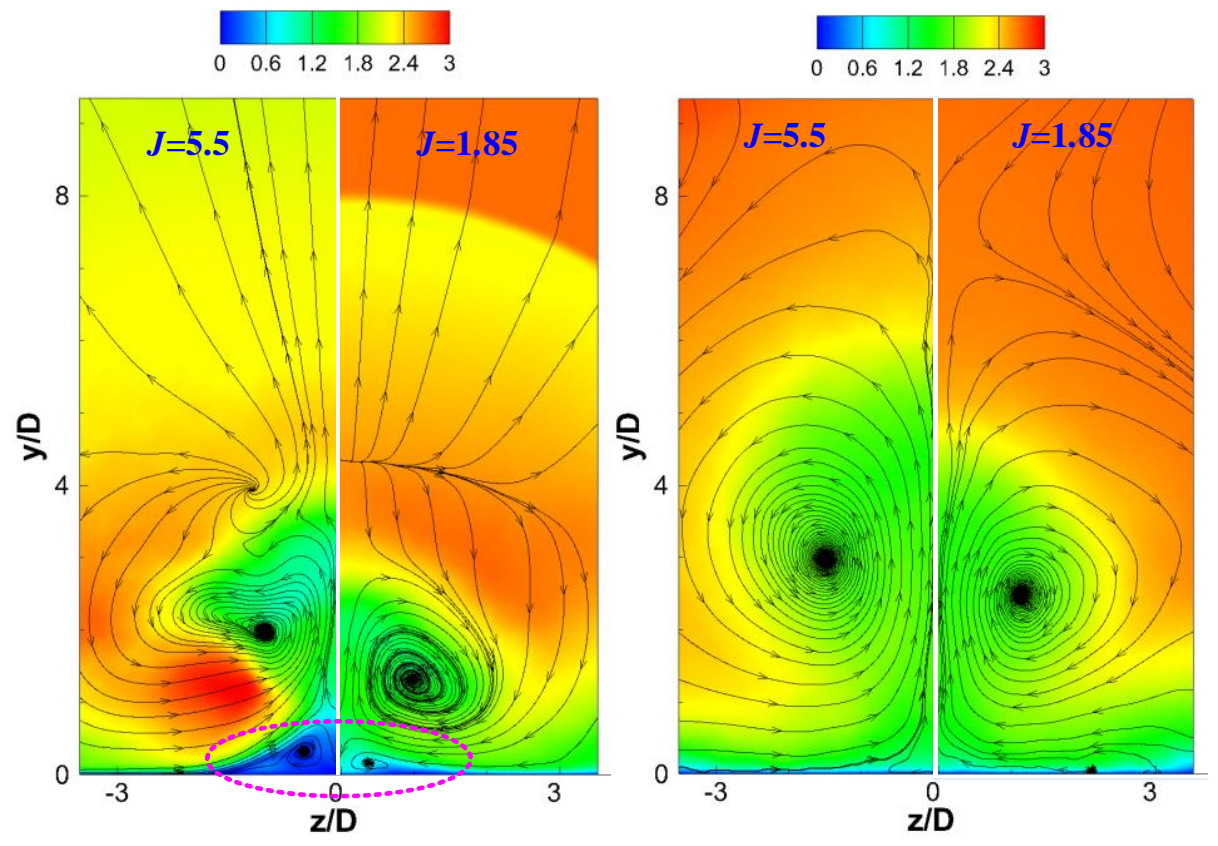

(c) $x / D=6.0$, Mach number contour

(d) $x / D=27.5$, Mach number contour

Fig. 9 Comparison of the time-averaged injectant mass fraction and Mach number contours with local in-plane streamlines between $J=1.85$ and $J=5.5$ on cross sections of $x / D=6.0$ and 27.5 .

\subsection{Three-dimensional streamline analysis}


Transport of the injectants towards the wall in the jet nearfield and farfield is further analyzed in this section using 3D streamlines to demonstrate the injactant transport process and the resulted distribution in the nearwall zone.

Fig.10 presents an oblique view and the corresponding front view of the flowfield containing the streamlines originating from the inside of the jet orifice and the streamlines aiming to the nearwall position at the exit for $J=1.85$. The streamlines are marked with spheres coloured by the local time-averaged injectant mass fraction. The $J=5.5$ case shows similar topology and therefore it is not given here. In Fig.10 (a) and (c) the streamlines exhibit significant curvatures, which indicate the effects of the major CVP. Contours of the time-averaged injectant mass fraction on the slice of $x / D=40.0$ give an indication of the extent of the injectant transport. Fig.10 (a) and (c) show streamlines originating from the jet orifice and indicate the motion and mixing of the jet fluid. The streamlines originating in the jet orifice follow the major CVP into the core of the jet plume. A close observation indicates that the major CVP generally pumps the injectant away from the wall. In the farfield, the streamlines follow the lifting and rotating major CVP and bring the injectants to a higher position away from the nearwall region. From an overall view, the streamlines inherit the rotating movement from the nearfield while they have little deformation or twist and go directly downstream in the farfield.

Fig.10 (b) and (d) show the representative streamlines of $J=1.85$ traced back from farfield nearwall positions at $y / D=0.5$ and $y / D=1.0$ on $x / D=40.0$ slice. It is seen that those streamlines originate from the crossflow in the lateral side running around the jet body. Only a small amount of the injectant in the nearwall region is entrained to the farfield. Streamlines run around the jet body and gain injectants when penetrating the injectant rich region. Streamlines in Fig.10(b) do not exhibit strong 
rotating motion since the intensity of major CVP greatly decreases in the lateral side of the jet plume. Ferrante et al. ${ }^{[23]}$ conducted LES to study a helium sonic jet in supersonic crossflows and found almost no helium near the wall, whereas the experiments showed a non-zero mole fraction of helium below the main core of the jet. They attributed this to the absence of a wall-normal mass flux model in their simulations. In the current simulation, the detailed turbulence and jet fluid mixing is well resolved and the injectant distribution in the nearwall region is clearly identified, without necessity to conduct flux models on the wall.

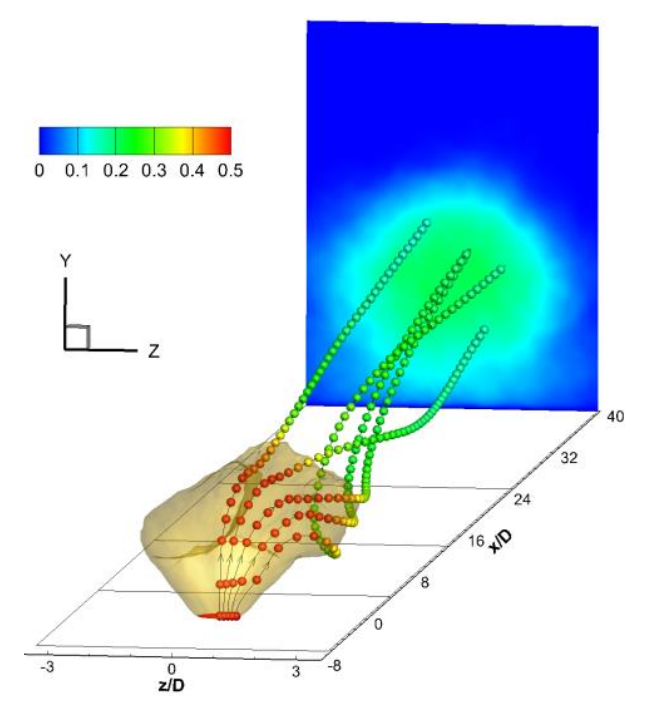

(a) 3D streamlines originating from jet orifice, oblique view

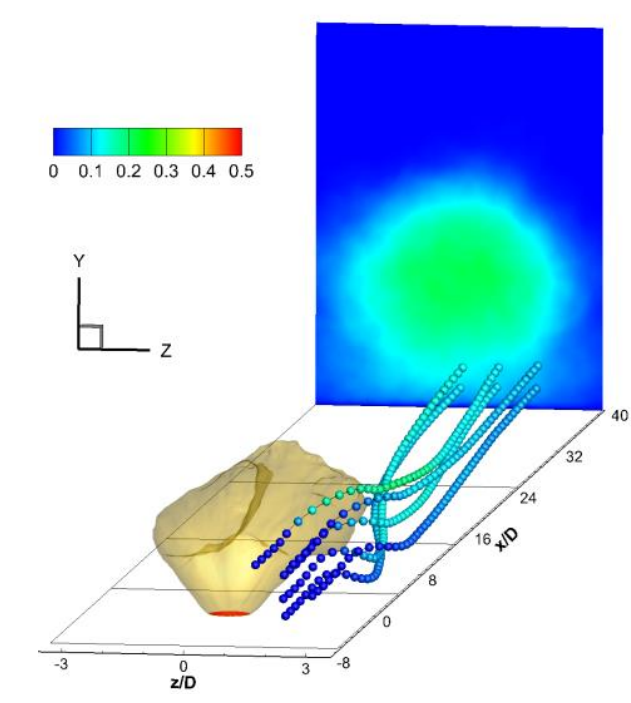

(b) 3D streamlines aiming at $y / D=0.5$

and 1.0 (nearwall region) at the exit, oblique view 


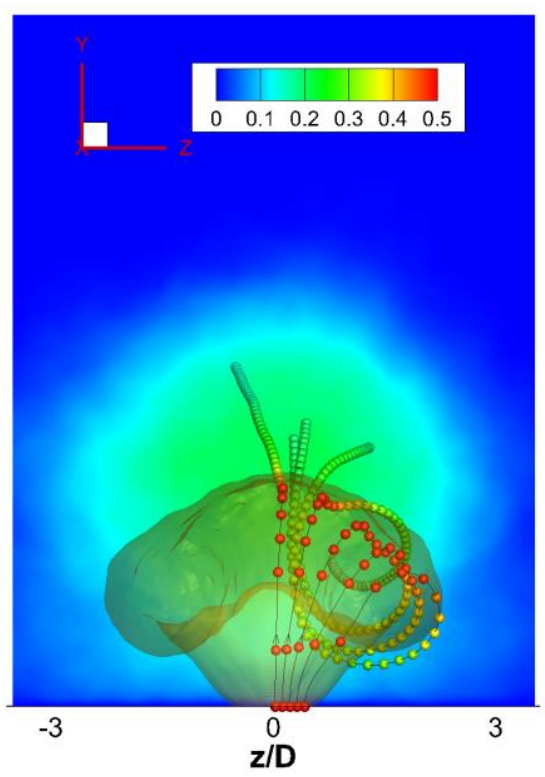

(c) 3D streamlines originating from jet orifice, front view

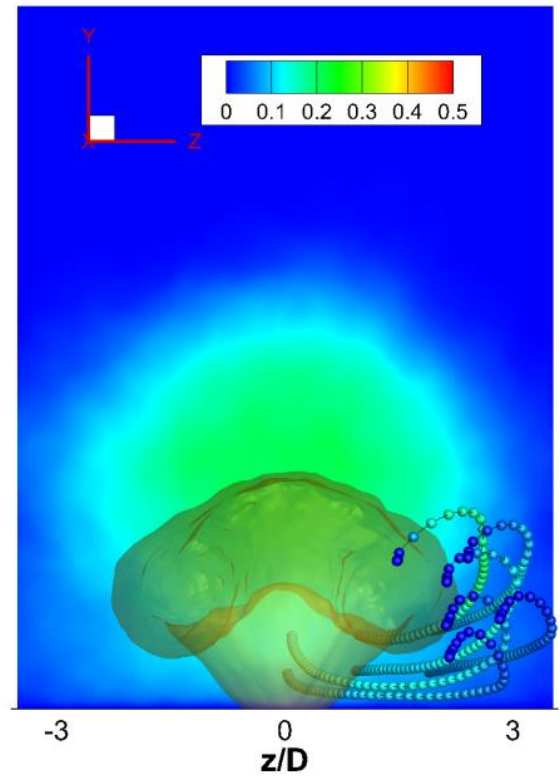

(d) 3D streamlines aiming at $y / D=0.5$ and 1.0 (nearwall region) at the exit, front view

Fig.10 3D streamlines originating inside the jet orifice and the streamlines aiming to the nearwall position on the exit of $J=1.85$, colored by local injectant mass fraction. Iso-surface of $Y_{\mathrm{i}}=0.4$ is also given.

To more clearly illustrate the injectant entrainment to the nearwall region, Fig.11 give the streamlines passing the lines at $x / D=-6.0,6.0,19.0$ and 32.0 on the plane of $y / D=0.5$. It exhibits that the streamlines originating from the lateral side of the crossflow run around the jet body and approach the nearwall region in the nearfield and farfield. These streamlines, colored by the injectant mass fraction, show that part of the jet fluid, especially the injectant adjacent to the jet orifice, is entrained and mixed with the air stream.

Fig.11(a) show the representative streamlines which originate from the crossflow at $y / D=0.5$ and $x / D=-6.0$ upstream of the jet. It is seen that some of the injectants is 'trapped' in the downside region of the jet owing to the major CVP and the lower fluid velocity in the center of the jet lee. This phenomenon explains the upwash 
velocity under the jet plume in the $2 \mathrm{D}$ symmetric slice $z / D=0.0$ of Fig.5(b). Streamlines in the jet lateral are found to entrain the injectant to the nearwall region in the farfield. In Fig.11(b), representative streamlines, which pass the nearwall position at $y / D=0.5$ and $x / D=6.0$, come from the lateral side of the crossflow and wrap around the jet orifice. The streamlines near the jet orifice concentrate in the jet lee and move to a higher position due to the upwash. Some of the streamlines coming from the lateral crossflow run into the nearwall region downstream. Streamlines passing $y / D=0.5$ plane in the farfield, shown in Fig.11(c) and (d), wholly come from the crossflow in the jet lateral and gain a downwash velocity to approach the wall in the farfield. In Fig.11(c), the streamlines running around the jet orifice are affected slightly by the upwash flow in the farfield. In Fig.11(d), streamlines in the farfield demonstrate that the injectants in the nearwall region come from the entrainment of the lateral crossflow.

As a summary of the analysis of Fig.10 and Fig.11, the streamlines originating from the jet orifice follow the major CVP and penetrate into the core of the jet plume. The jet fluid transported to the nearwall region downstream of the jet originates primarily from the crossflow in the upstream lateral of the jet, which runs around the jet orifice and gain a downwash velocity to approach the wall in the farfield. 


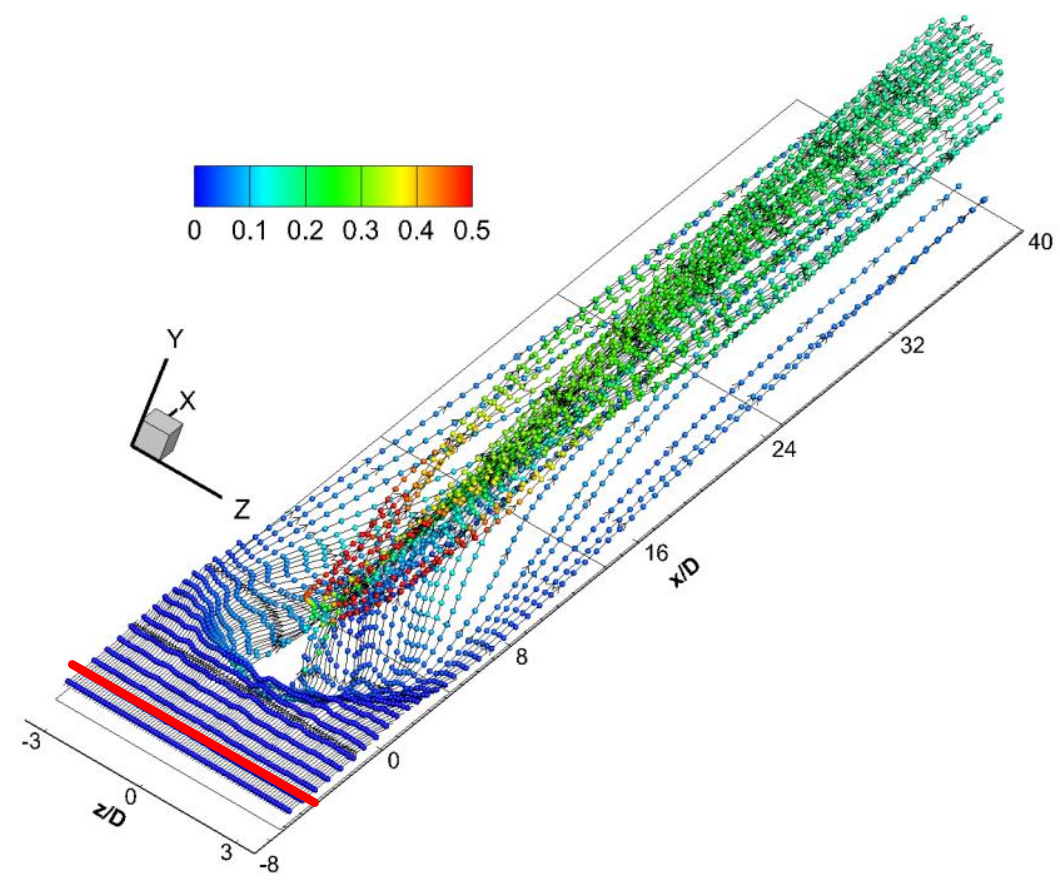

(a) Streamlines passing the wall parallel plane $y / D=0.5$ at $x / D=-6.0$ (Red line)

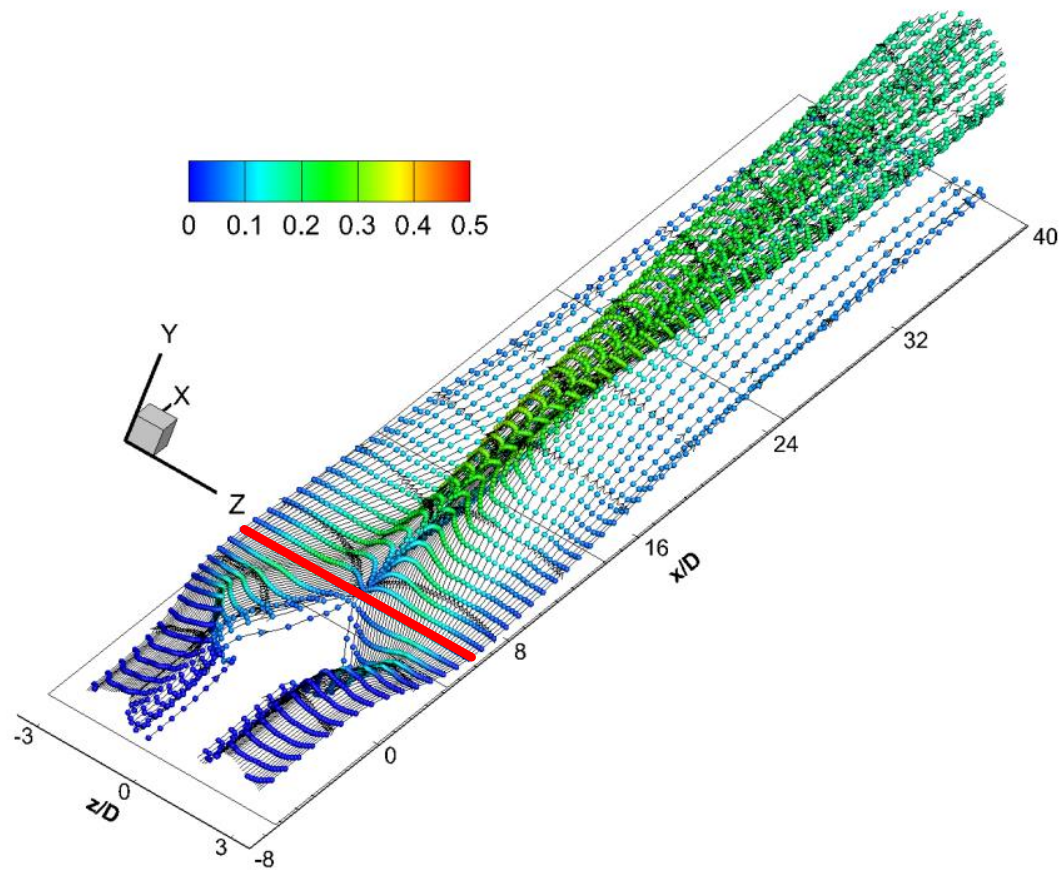

(b) Streamlines passing the wall parallel plane $y / D=0.5$ at $x / D=6.0$ (Red line) 


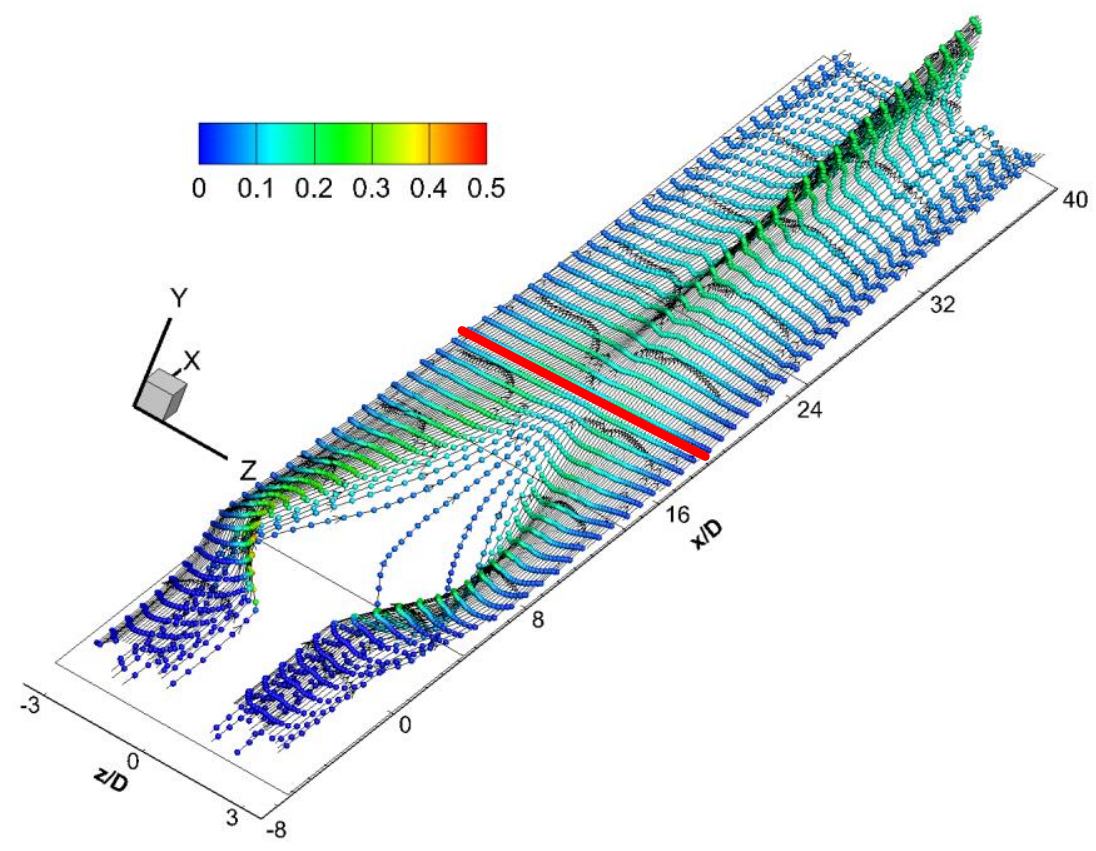

(c) Streamlines passing the wall parallel plane $y / D=0.5$ at $x / D=19.0$ (Red line)

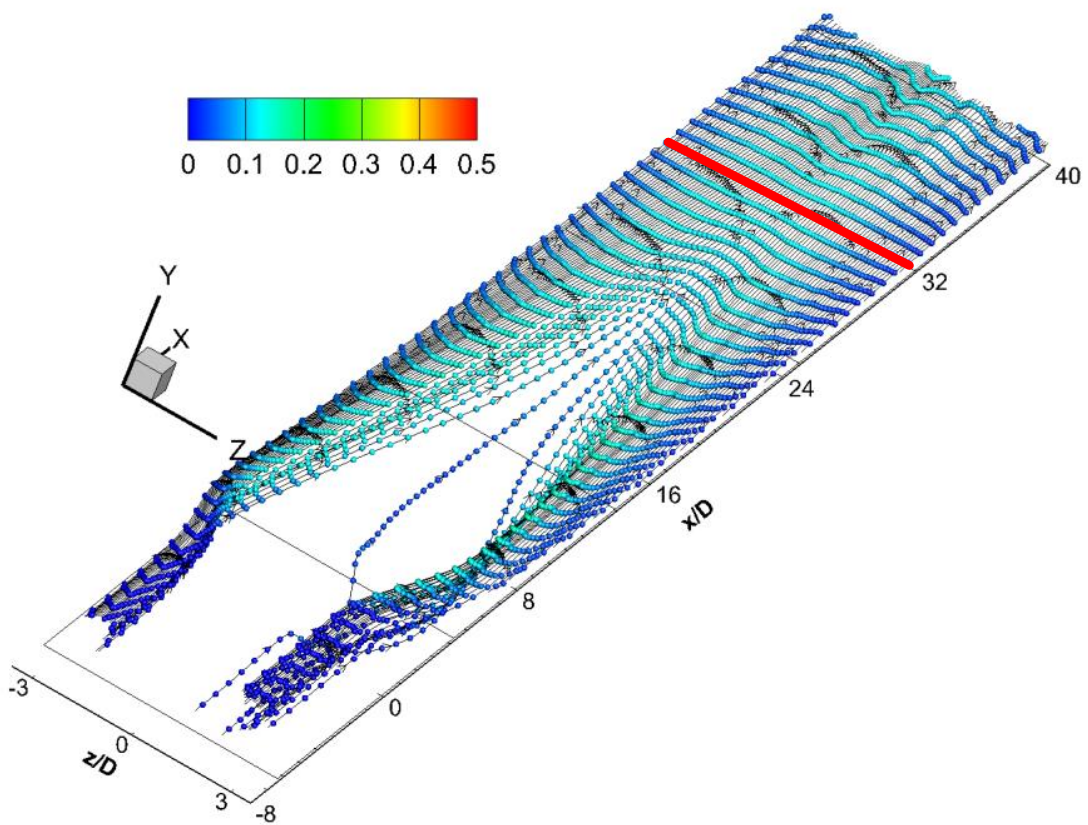

(d) Streamlines passing the wall parallel plane $y / D=0.5$ at $x / D=32.0$ (Red line)

Fig.11 Oblique view of $3 \mathrm{D}$ streamlines for $J=1.85$ passing through the lines on the wall-parallel plane $y / D=0.5$ at different streamwise locations, colored by the injectant mass fraction.

\subsection{Injectant distribution in the nearwall region downstream of the jet orifice}

In this section we will discuss the injectant mass distribution to quantify the 
qualitative conclusions presented in the previous sections. Profiles of the timeaveraged injectant mass fraction are shown in Fig.12(a) at different streamwise locations. In the jet nearfield, the injectant mass fraction of $J=5.5$ is smaller than $J=1.85$ for $y / D<2.0$ (indicated by the blue dashed line in Fig.12a), which strongly suggests that in the jet nearfield more injectant is entrained into the nearwall zone at lower $J$. On the contrary, in the jet farfield, also shown for $x / D=27.5$ and 40.0 in Fig. 12(a), the injectant mass fraction of $J=5.5$ is always larger than that of the $J=1.85$ case. This contradicts the suggestion given by previous research ${ }^{[25]}$, which considered a local injectant lean result of the incoming flow and attributed the downstream extinction to the fuel-lean status.

Time averaged streamwise velocity distributions for $J=1.85$ and $J=5.5$ are shown in Fig.12(b). It is seen that at the outlet of $x / D=40.0$, the profiles of the streamwise velocity remain similar for the two cases with a small deformation seen for $J=5.5$ due to the stronger interaction. Based on Fig.12, for the explanation of the experiments in $\operatorname{Re}{ }^{[25]}$, the current simulation reveals that a local injectant-rich status in the nearwall farfield is possible to result in the flame extinction when $J$ varies from 1.6 to 2.5 in the farfield (where $x / D>120.0$ in $\operatorname{Ref}^{[25]}$ ).

As well known, jet plume has a 3D configuration and the mass fraction proflies in the mid-span $z / D=0.0,3.45$ are not enough to describe the injectant distribution. Fig. 13 gives a comparison of the mean injectant mass fraction profiles of $J=1.85$ and $J=5.5$ over the spanwise direction at different streamwise locations. Firstly, it is seen that close to the symmetric line $z / D=0.0$ in the nearfield $(x / D=8.5$ and 15.0$)$, the injectant mass fraction is always lower than the peak of the same profile for both cases. The peak corresponds to the core of the major CVP. Therefore, a 'gap' with a low injectant mass fraction between the CVP branches is expected. Secondly, in the 
farfield $(x / D=27.5$ and 40.0), the injectant mass fraction near $z / D=0.0$ is approximately equal to the peak value of the same profile, which indicates a mixing and smeared effect with the development of the major CVP.

The injectant mass fraction of $J=1.85$ at $y / D=1.0$ and $x / D=8.5$ is larger than that of $J=5.5$ over the spanwise region of $z / D \in[0,2.15]$, which is due to the higher penetration and the larger 'gap' between the major CVP branches for $J=5.5$. While over $z / D \in[2.15,3.5]$, the injectant mass fraction of $J=1.85$ is smaller than $J=5.5$, since higher $J$ would bring more injectants into the core of the major CVP. At $y / D=2.0$ and $x / D=8.5$, over the spanwise region of $z / D \in[0,1.25]$, the injectant mass fraction of $J=1.85$ is higher than $J=5.5$ while it is smaller at $z / D \in[1.25,3.5]$, which indicates a decrease of the 'gap' width at a higher wall normal position. The gap between the CVP branches of $J=5.5$ lasts longer and has a longer distance than $J=1.85$, and that is why the injectant mass fraction of $J=5.5$ at $x / D=15.0$ is smaller near to the symmetric plane, as shown in Fig.13(b). The low mass fraction zone near to the symmetric plane increases with $J$, which corresponds to an extension of Region I, as analyzed in Section 3.2. In Fig.13(c) and (d) at $x / D=27.5$ and 40.0, the injectant mass fraction of $J=5.5$ is higher than $J=1.85$ across the whole span, which means that after the recovery of Region I, more injectant is entrained into the nearwall region in the farfield for higher $J$.

Fig.14 show the mass fraction distribution on four representative lines of $J=5.5$ and $J=1.85$ along the $x$-axis. Injectant mass fraction on these lines clearly reflects the phenomenon that in the nearfield a low mass fraction zone exists. At $y / D=1.0$, mass fraction in Region I of $J=1.85$ is larger than $J=5.5$ and it is still true at $y / D=2.0$ and $z / D=0.0$. However, at $y / D=2.0$ and $z / D=2.0$, this phenomenon disappears since at 
$x / D>0.0$ injectant mass fraction of $J=5.5$ is always larger than $J=1.85$. This reveals the 3D configuration of Region I.
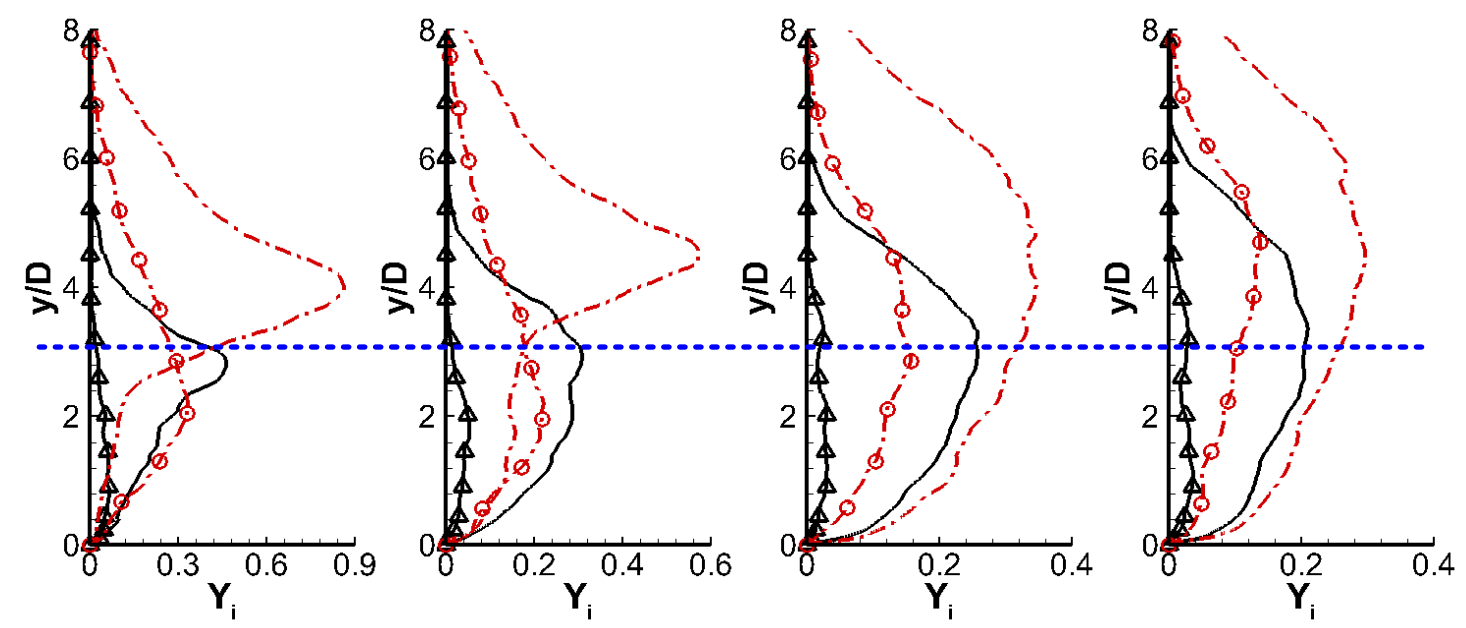

(a) Time averaged injectant mass fraction profiles of $J=1.85$ (solid line) and $J=5.5$

(dashdotted line), $x / D=8.5,15,27.5,40$ (from left to right), respectively
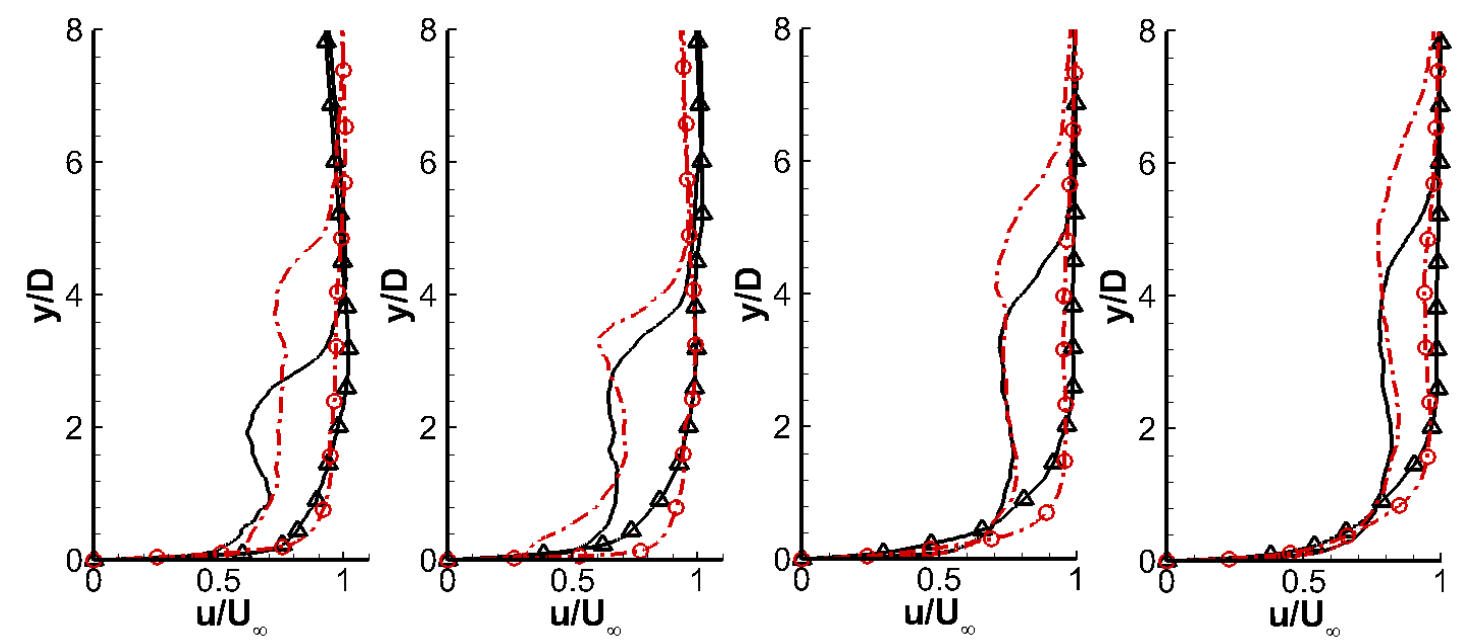

(b) Time averaged streamwise velocity profiles of $J=1.85$ (solid line) and $J=5.5$ (dashdotted line), $x / D=8.5,15,27.5,40$ (from left to right), respectively

Fig. 12 Comparisons of injectant mass fraction (a) and time averaged streamwise velocity (b) of $J=1.85$ and $J=5.5$ at $x / D=8.5,15,27.5,40$ (from left to right), respectively. Solid lines: $J=1.85, z / D=0$; Dashdotted line: $J=5.5, z / D=0$; Solid line with triangle $(\Delta): J=1.85, z / D=3.45$; Dashdotted line with circle $(\circ): J=5.5, z / D=3.45$. 


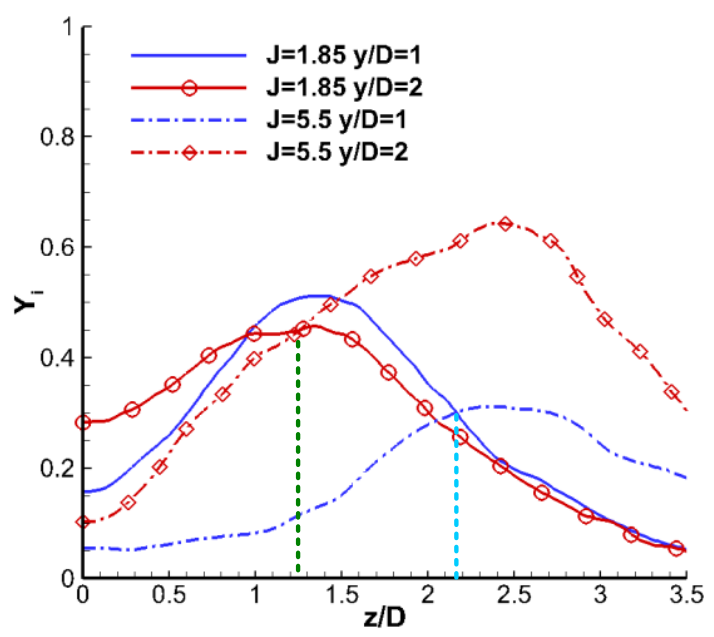

a) $x / D=8.5$

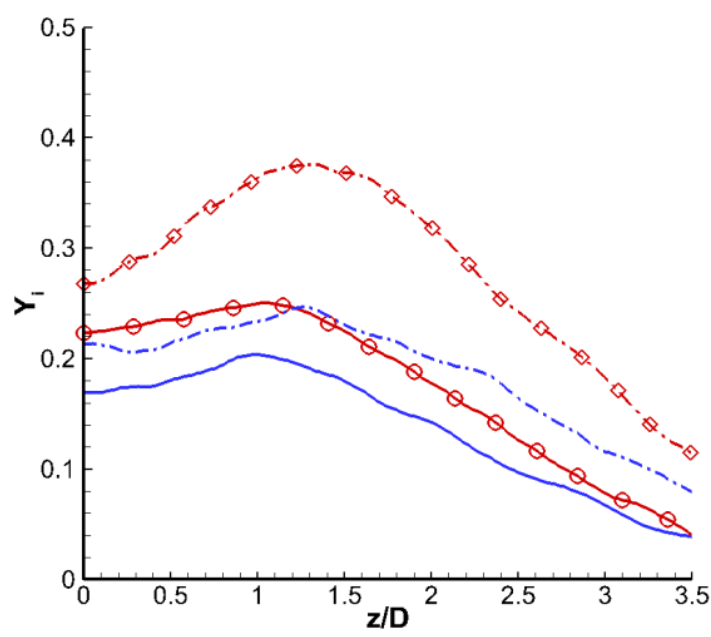

c) $x / D=27.5$

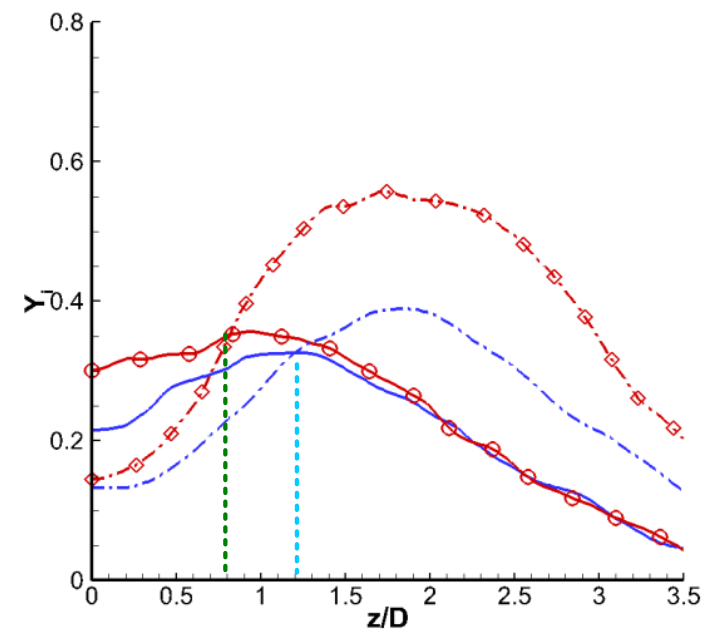

b) $x / D=15.0$

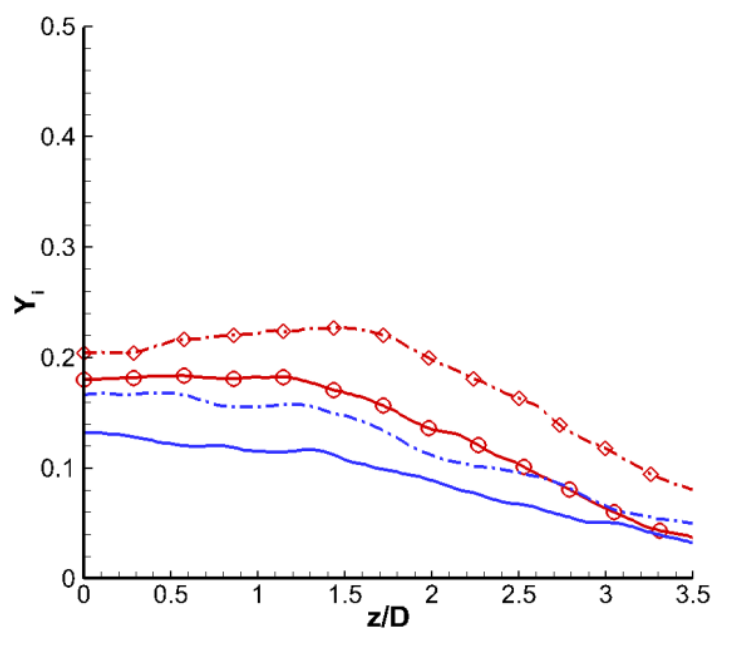

d) $x / D=40.0$

Fig. 13 Comparisons of mean injectant mass fraction of $J=1.85$ and $J=5.5$ at $y / D=1.0$ and 2.0 at different streamwise locations, Solid lines: $J=1.85, y / D=1.0$; Dashdotted line: $J=5.5, y / D=1.0$; Solid line with circle $(\circ): J=1.85, y / D=2.0$; Dashdotted line with diamond $(\diamond): J=5.5, z / D=2.0$. 


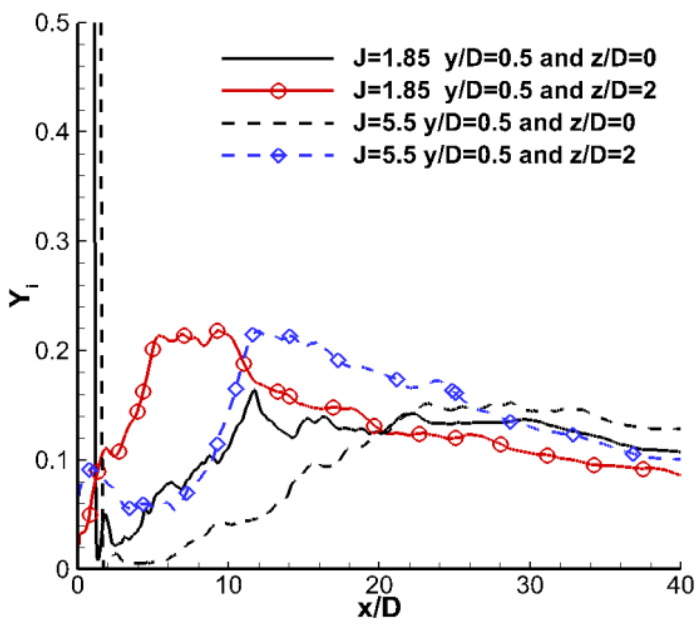

(a) $y / D=0.5$

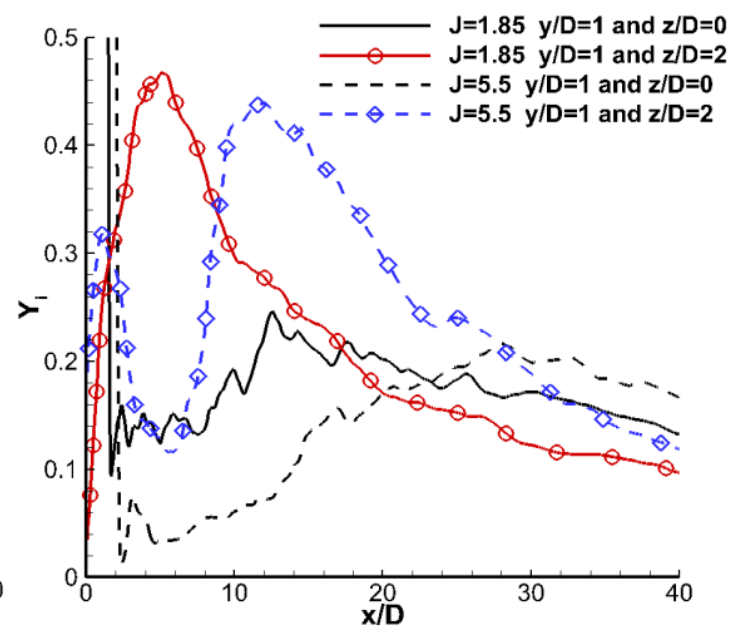

(b) $y / D=1.0$

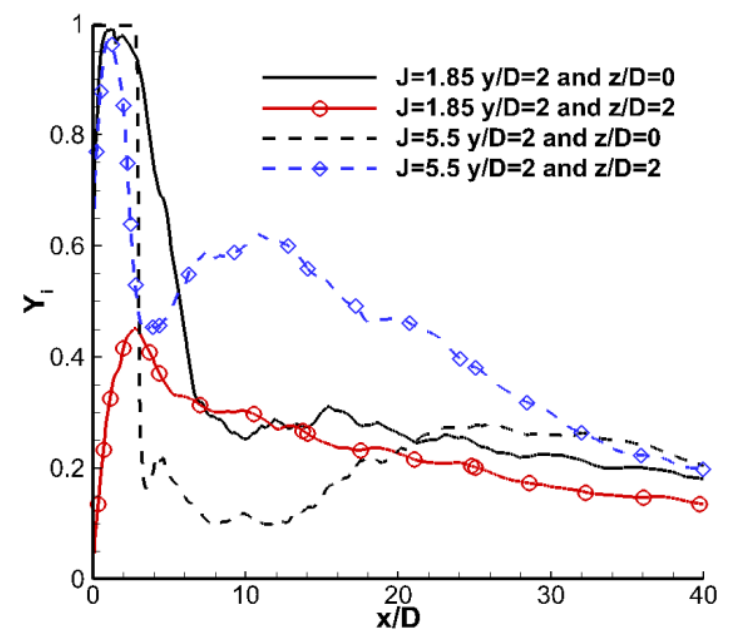

(c) $y / D=2.0$

Fig.14 Comparisons of the time-averaged injectant mass fraction of $J=1.85$ and $J=5.5$ at $y / D=0.5,1.0$ and $2.0, z / D=0.0$ and 2.0 along the streamwise direction

The results shown in Figs.12-14 demonstrate that the jet with lower $J(=1.85)$ leads to a higher injectant concentration in the nearwall region located in the jet nearfield (Region I), but produces a lower concentration in the farfield. To better describe the mixing characteristics of the injectant distribution, the area excluding Region I is defined as Region II, as shown in Fig.15. In Region I, there exists two zones. One zone is associated with the nearwall separation in the jet lee. The other 
zone is associated with the gap between the plume CVP branches. Region I expands as $J$ becomes larger and less injectant is entrained into Region I, which explains the decrease of the injectant mass fraction with an increased $J$ in this region. On the contrary, the injectant mass fraction in Region II increases with $J$ since higher $J$ brings more injectants. In the farfield of Region II, the injectant is entrained into the nearwall zone and has a higher mass fraction magnitude when larger $J$ is set. From a direct point view, Region I is thought to be caused by the blockage of the jet body in the supersonic crossflow. Especially when $J$ becomes higher, the jet body expands to a larger one. When mixing enhancement is concerned in the nearfield, it is useful to reduce the jet blockage in the supersonic crossflow, which might lead to jet arrays or staged injection $^{[3]}$. Fig.15 only gives a 2D schematic and in the next section we will discuss the 3D configuration of Region I and Region II.

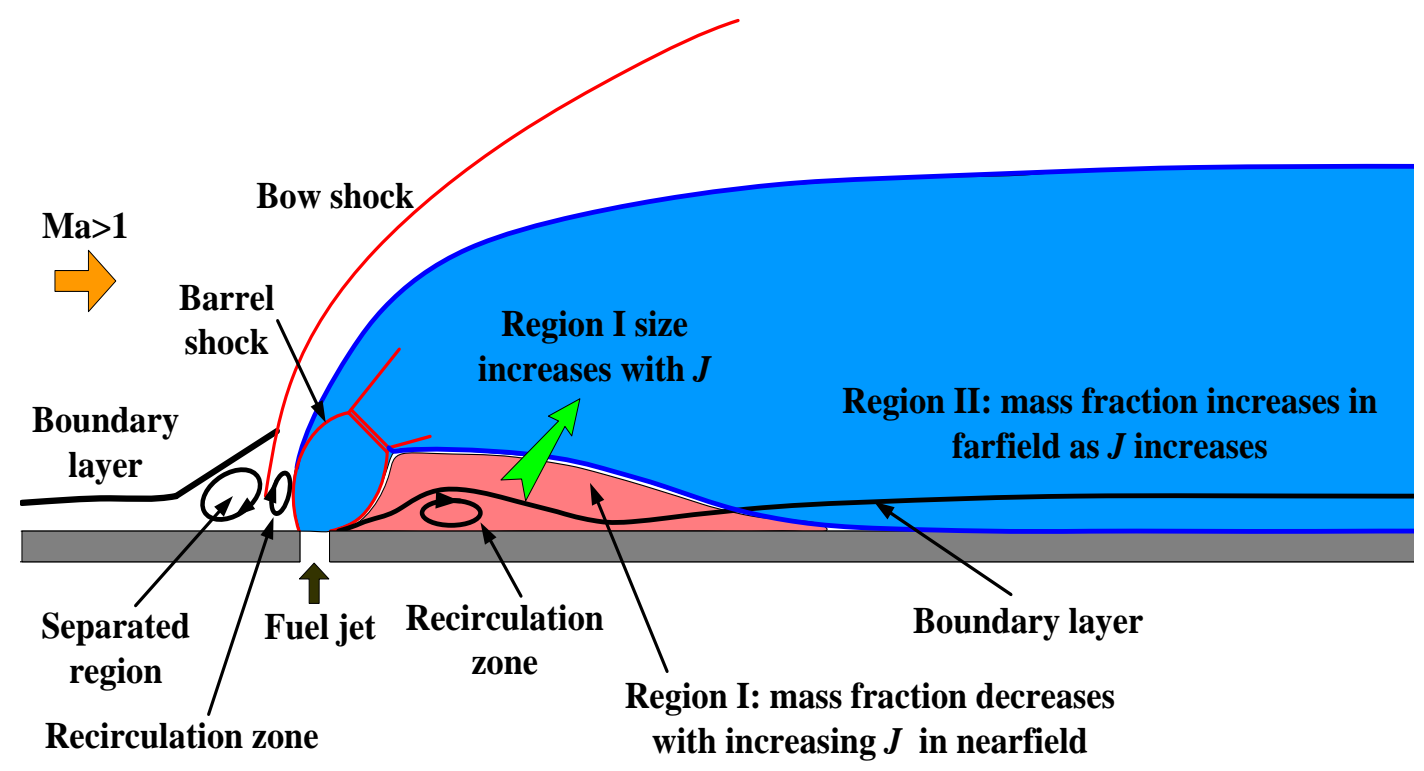

Fig.15 Schematic of the mass fraction changes in Region I and II with increasing $J$

\subsection{Three-dimensional configuration of Region I and II}

In this section further detailed analysis is given to explain the Region I and II configurations. Fig.16 shows the iso-surface of the injectant mass fraction $Y_{\mathrm{i}}=0.1$ for 
$J=1.85$, which indicates the configuration of Region II since Region I is under the jet plume near the wall. As discussed in Section 3.3-3.4, Region I is near to the wall in the jet nearfield. Region II includes the major CVP plume region and the nearwall zone in the farfield.

To clearly show Region I, the iso-surface is shown with a surface cut through which we can see the jet nearfield nearwall configuration. By conducting a slice cut on the $Y_{\mathrm{i}}$ iso-surface, iso-surfaces of $Y_{\mathrm{i}}=0.1$ and 0.05 of $J=5.5$ and $J=1.85$ are shown in Fig.17(a)-(b), respectively. The dotted circle marks the skeleton of Region I in the jet lee. Fig.17(a) demonstrates that Region I has a bump with a low mass fraction extending into the gap underneath the CVP branches. From the streamline analysis in section 3.4, streamlines run around the jet orifice and little injectant is entrained into Region I. In Fig.17(c), iso-surfaces of the streamwise velocity $u / U_{\infty}=0.0$ of $J=5.5$ and $J=1.85$, colored by the local wall-normal distance, are shown to demonstrate the $3 \mathrm{D}$ near-wall separation configuration. Further downstream of the jet leeward separation bubble exist two separate separation wings. The peak of the oblique wing corresponds to the edge of the separation zone. Comparing $J=5.5$ with $J=1.85$, we can see that the lower $J$ case has a similar character of flow configuration, although the size is smaller than $J=5.5$. Detailed formation mechanism about the separation bubble can be found in our recent work ${ }^{[22]}$ which gave the detailed structures of the separation interaction with the collision shock and the induced surface trailing CVPs in the jet wake. From Fig.17(c) it is seen that the separation zone is fully 3D which re-defines the separation zone in Region I shown in Fig.15.

Fig.18 gives the 3D configurations of Region I and Region II based on the above analysis. Region I locates under the jet plume near the wall, containing the jet leeward separation zone and the gap between the plume CVP branches. In the farfield of 
Region II, the jet plume expands and approaches the wall, which leads to a mass fraction increase in the nearwall region of Region II. The reattachment line on the wall also represents the end of Region I. As analyzed above, higher $J$ leads to an expansion of Region I, which results in a lower injectant concentration in Region I, but produces a higher concentration in the farfield (Region II Farfeild). As a summary, when $J$ increases, the change of the injectant concentration or mass fraction varies near the wall along the streamwise direction. In Region I, the change of the injectant concentration or mass fraction is opposite to the $J$ change, while in the nearwall zone of Region II, the change of the injectant concentration or mass fraction is synchronous with $J$ change. This simulation clearly clarifies the dispute whether higher $J$ would increase the downstream nearwall injectant concentration or not.
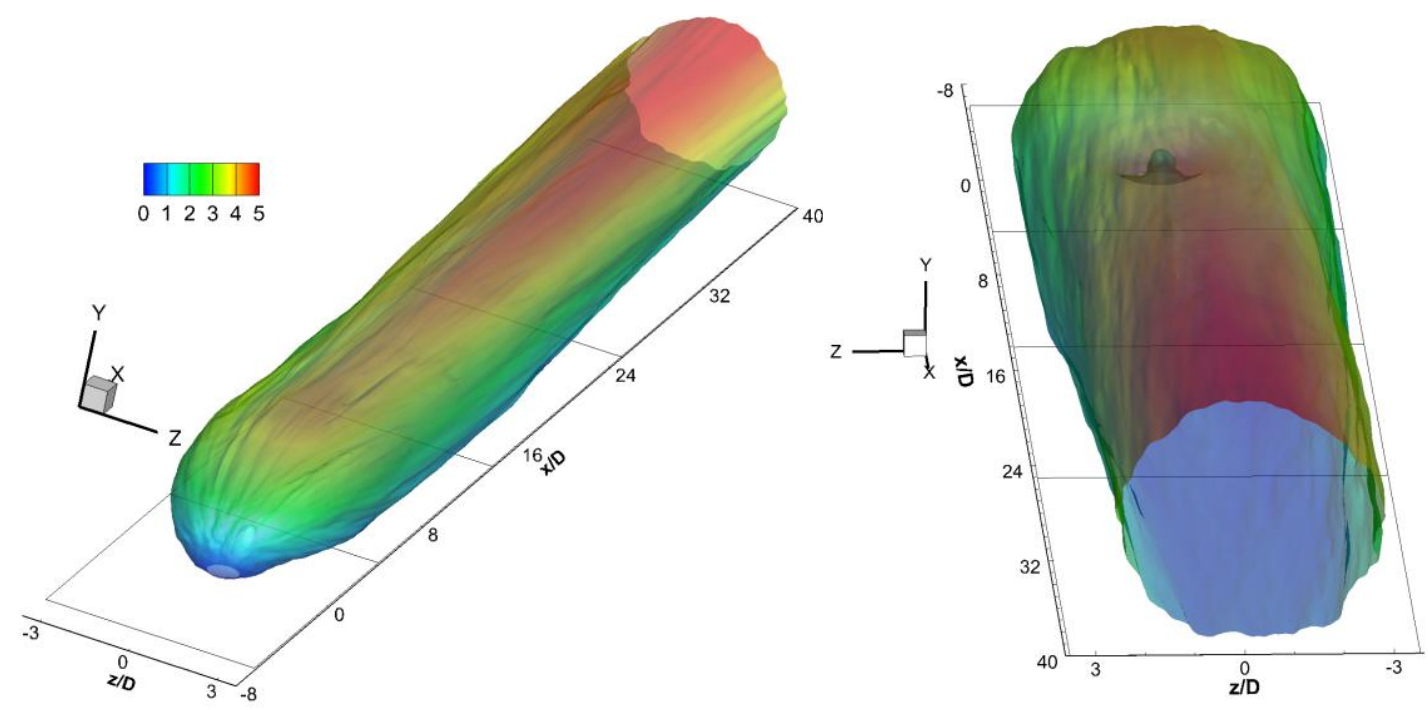

Fig.16 Transparent iso-surface of the injectant mass fraction $Y_{\mathrm{i}}=0.1$ of $J=1.85$, indicating Region II, colored by the wall normal distance. Oblique view (left) and back view (right). 

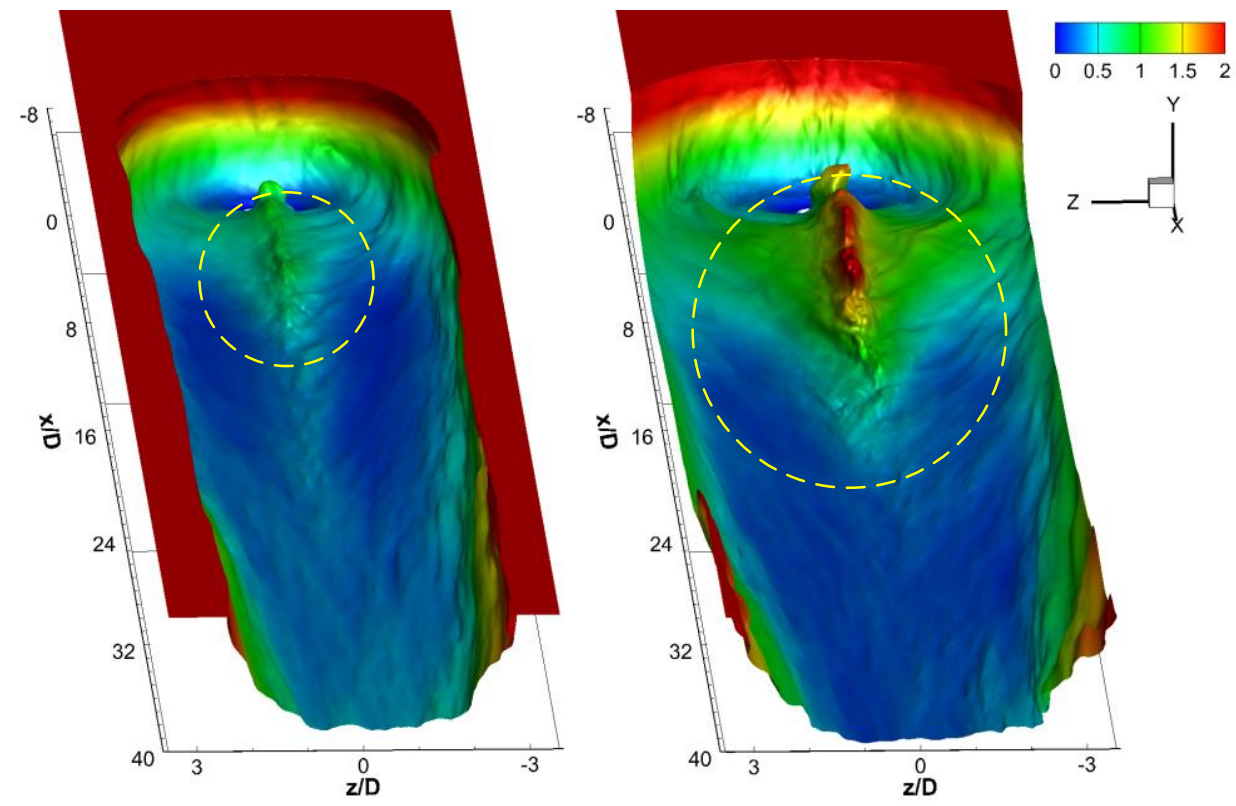

(a) Iso-surface of the injectant mass fraction $Y_{\mathrm{i}}=0.1$ near the wall with a slice cut view, from left to right $(J=1.85$ and $J=5.5)$
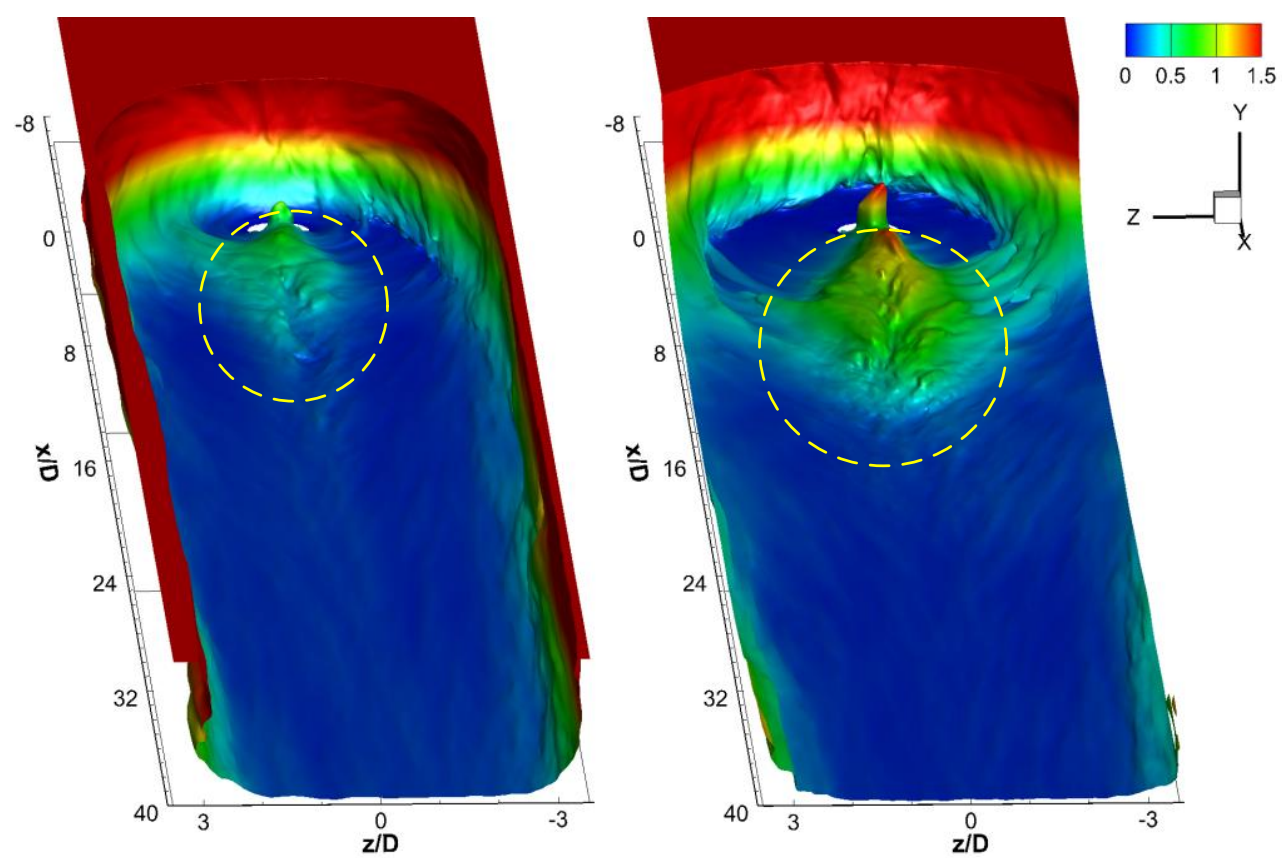

(b) Iso-surface of the injectant mass fraction $Y_{\mathrm{i}}=0.05$ near the wall with a slice cut view, from left to right $(J=1.85$ and $J=5.5)$ 

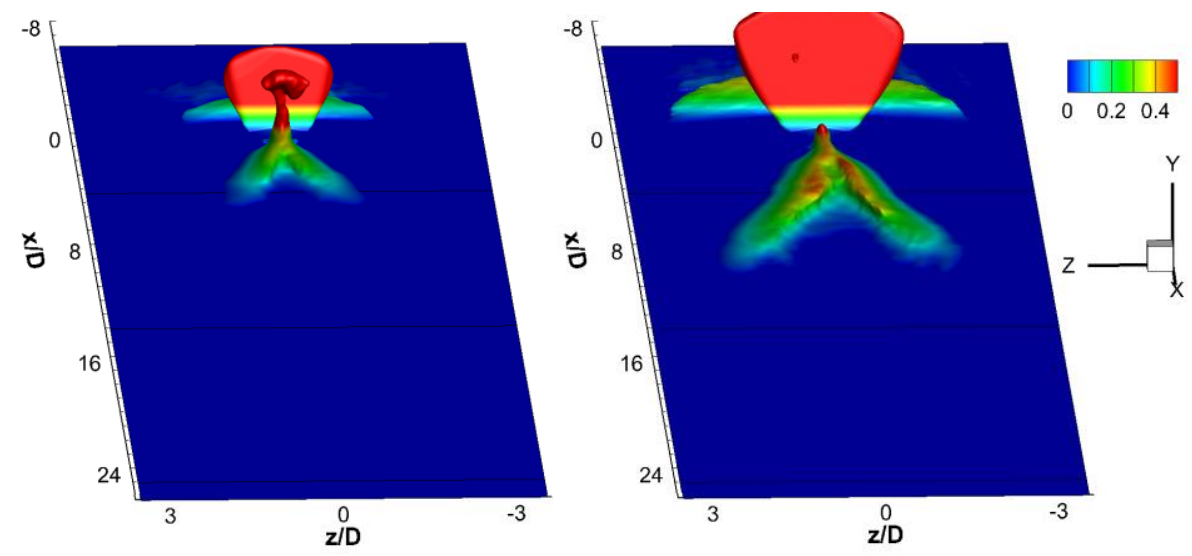

(c) Iso-surface of the streamwise velocity $u / U_{\infty}=0.0$ near the wall, representing the jet lee separation zone, from left to right $(J=1.85$ and $J=5.5)$

Fig.17 Iso-surface of the injectant mass fraction and streamwise velocity to show Region I in the jet leeward, colored by the wall normal distance

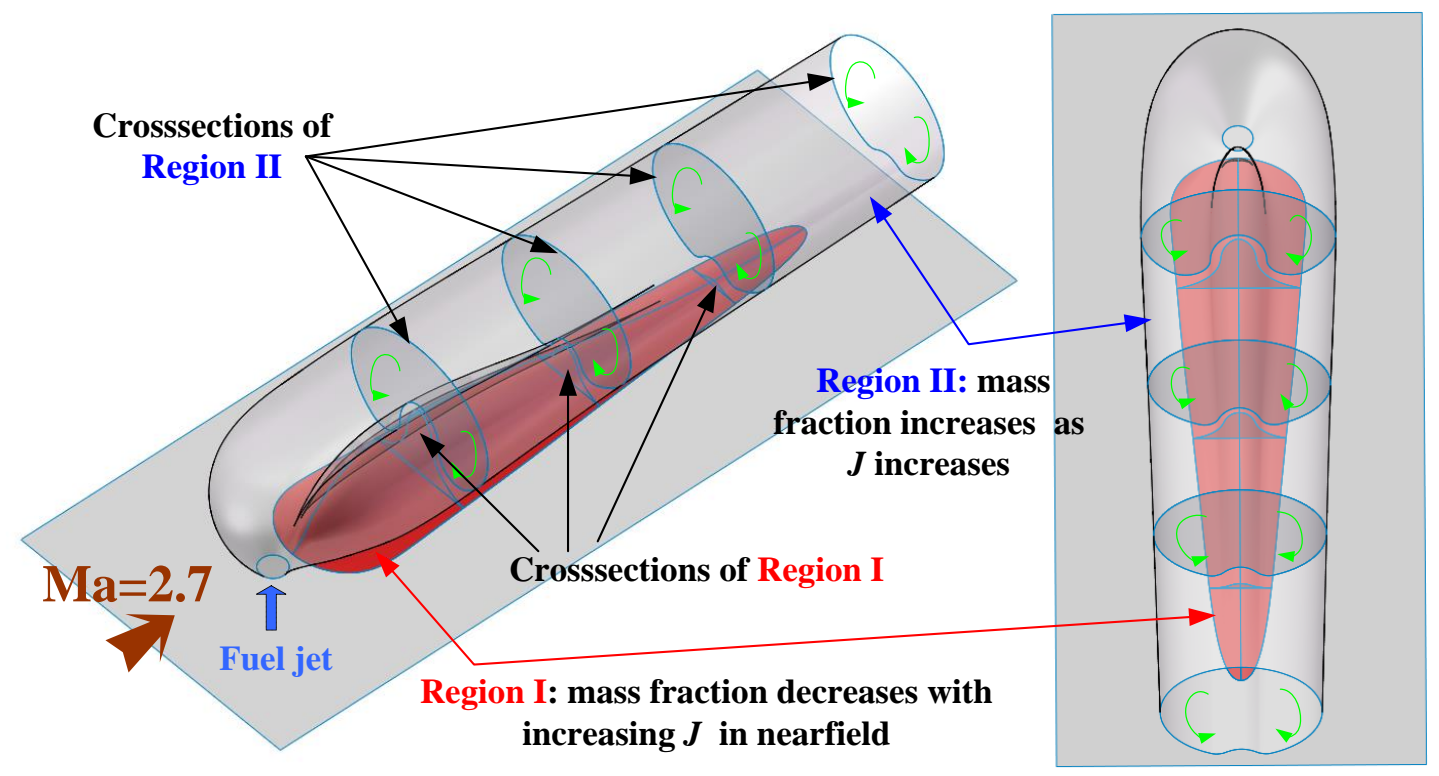

Fig.18 Schematic of the 3D configurations of Region I and II

\section{Conclusions}

In the present study, DNS is carried out to uncover nearwall mixing characteristics of a sonic jet injected into a supersonic crossflow at a Mach number of 2.7. Simulations are run for $J=1.85$ and 5.5 cases. The simulations provide 
instantaneous and time-averaged flow features including coherent structures, streamlines, mixing characteristics in the near-field and far-field downstream of the jet injection. The time-averaged jet penetrations are compared to the experimental correlations and good agreements are observed. It is shown that the large-scale major CVP dominates the nearfield mixing and break into smaller eddies in the farfield with an enhanced mixing effect. Analysis of the streamlines originating from the jet orifice suggests that the significant jet fluid entrainment can be attributed to the major CVP structures which pump the injectants into the core of the jet plume. Jet fluid in the nearwall region of the farfield is entrained by the streamlines originating from lateral crossflow, which travel around the jet and mix with the injectants downstream of the jet orifice.

Profiles of the injectant mass fraction are compared in details to quantify the distribution in the nearwall region downstream of the jet orifice. Detailed analysis exhibits that there are two regions downstream of the jet orifice, denoted as Region I and II. A 3D schematic is presented to explain the formation of Region I and II. Region I consists of the jet leeward separation zone and the gap between the plume CVP branches. Region II consists of the major CVP region and the nearwall region in the farfield. An injectant zone with low mass fraction exists in Region I. As $J$ becomes higher, the size of Region I increases and less injectant is entrained into the Region I, which leads to a decreased mass fraction zone in the nearfield. Region II increases in size as $J$ gets higher and meanwhile has a higher mass fraction in the farfield nearwall region. The current simulations reveal the variations of the nearwall injectant mass fraction with an increased $J$ along the streamwise direction, which clarifies the dispute whether higher $J$ would increase the nearwall injectant concentration or not. 


\section{Acknowledgement}

This work is funded by the National Science Foundation of China (grants: 11472305 and 11522222) and the outstanding youth fund of the National University of Defense Technology.

Sincere thanks to Professor N. D. Sandham of the University of Southampton for providing the SBLI (shock-/boundary-layer interaction) code and the helpful discussion on data analysis.

Supercomputer time provided by the UK Engineering and Physical Sciences Research Council under the project 'Thermal and Reactive Flow Simulation on HighEnd Computers' (Grant No. EP/J016381/1) is gratefully acknowledged.

\section{Reference}

[1] A. R. Karagozian. "Transverse jets and their control," Prog. Energy Combust. Sci. 36(5), $531-553(2010)$

[2] K. Mahesh. "The interaction of jets with crossflow," Annu. Rev. Fluid Mech. 45, 379-407 (2013)

[3] W. Huang, "Transverse jet in supersonic crossflows," Aerosp. Sci. Technol., 50, 183-195 (2016)

[4] A. Ben-Yakar, G. M. Mungal, and R. K. Hanson. "Time evolution and mixing characteristics of hydrogen and ethylene transverse jets in supersonic crossflows," Phys. Fluids, 18, 26-101 (2006)

[5] L. Maddalena, T.L. Campioli, J.A. Schetz, "Experimental and computational investigation of light-gas injectors in Mach 4.0 crossflow,” J. Prop. Power., 22(5), 1027-1038 (2006) 
[6] M. R. Gruber, J. M. Donbar, C. D. Carter, and K. Y. Hsu. "Mixing and Combustion Studies Using Cavity-Based Flameholders in a Supersonic Flow,” J. Propul. Power, 20 (5), 769-779 (2004)

[7] K. C. Lin, M. Ryan, C. Carter, M. Gruber, and C. Raffoul. "Raman Scattering Measurements of Gaseous Ethylene Jets in Mach 2 Supersonic Crossflow,” J. Propul. Power, $26(3), 503-514(2010)$

[8] T. Mai, Y. Sakimitsu, H. Nakamura, Y. Ogami, T. Kudo, and H. Kobayashi. "Effect of the incident shock wave interacting with transversal jet flow on the mixing and combustion," Proc. Combust. Inst., 33, 2335-2342 (2011)

[9]W. M. VanLerberghe, J. G. Santiago, J. C. Dutton, R. P. Lucht. Mixing of a sonic transverse jet injected into a supersonic flow, AIAA J, 2000, 38(3):470-79

[10] M. B. Sun, H. Geng, J. H. Liang, and Z. G. Wang. "Mixing Characteristics in a Supersonic Combustor with Gaseous Fuel Injection Upstream of a Cavity Flameholder," Flow Turbul. Combust., 82, 271-286 (2009)

[11] M. B. Sun, S. P. Zhang, Y. H. Zhao, Y. X. Zhao, and J. H. Liang. "Experimental investigation on transverse jet penetration into a supersonic turbulent crossflow," Sci. China Tech. Sci., 56 (8), 1989-1998 (2013)

[12] S. Kawai, and S. K. Lele. "Large-Eddy Simulation of Jet Mixing in Supersonic Crossflows, ”AIAA J., 48 (9), 2063-2083 (2010)

[13] Z. A. Rana, B. Thornber, and D. Drikakis. "Transverse jet injection into a supersonic turbulent cross-flow,” Phys. Fluids, 23 (046103) (2011)

[14] S. H. Won, I. S. Jeung, B. Parent, and J. Y. Choi. "Numerical Investigation of Transverse Hydrogen Jet into Supersonic Crossflow Using Detached-Eddy Simulation,” AIAA J., 48 (6), $1047-1058(2010)$

[15] Y. You, H. Luedeke, and K. Hannemann. "Injection and mixing in a scramjet combustor: DES and RANS studies,” Proc. Combust. Inst., 34, 2083-2092 (2013)

[16] J. A. Boles, J. R. Edwards, R. A. Baurle. Large-eddy/Reynolds-averaged Navier-Stokes simulations of sonic injection into Mach 2 crossflow. AIAA J. 48(7), 1444-56 (2010) 
[17] E. Hassan, J. Boles, H. Aono, D. Davis, W. Shyy, Supersonic jet and crossflow interaction: Computational modeling, Prog. Aerosp. Sci., 57, 1-24 (2013)

[18] X. Chai, P. S. Iyer, and K. Mahesh. "Numerical study of high speed jets in crossflow," J. Fluid Mech., 785, 152-188 (2015)

[19] H. B. Wang, Z. G. Wang, M. B. Sun, and N. Qin. "Hybrid Reynolds-averaged NavierStokes/large-eddy simulation of jet mixing in a supersonic crossflow," Sci. China Tech. Sci., 56 (6), 1435-1448 (2013)

[20] Y. J. Zhang, W. D. Liu, and M. B. Sun. "Effect of microramp on transverse jet in supersonic crossflow," AIAA J., 54 (12), 4041-4044 (2016)

[21] M. B. Sun, and Z. W. Hu. "Generation of Upper Trailing Counter-Rotating Vortices of a Sonic Jet in a Supersonic Crossflow," AIAA J, 56 (3), 1047-1059 (2018)

[22] M. B. Sun, and Z. W. Hu. "Formation of surface trailing counter-rotating vortex pairs downstream of a sonic jet in a supersonic crossflow,” J. Fluid Mech., 850, 551-583 (2018)

[23] A. Ferrante, G. Matheou, and P. E. Dimotakis. "LES of an inclined sonic jet into a turbulent crossflow at Mach 3.6," J. Turbul., 12 (2), 1-32 (2011)

[24] Z. G. Wang, M. B. Sun, H. B. Wang, J. F. Yu, J. H. Liang, and F. C. Zhuang. "Mixingrelated Low Frequency Oscillation of Combustion in an Ethylene-Fueled Supersonic Combustor,” Proc. Combust. Inst., 36 (2), 2137-2144 (2015)

[25] M. B. Sun, X. D. Cui, H. B. Wang, and V. Bychkov. "Flame flashback in a supersonic combustor with ethylene injection upstream of cavity flameholder," J. Propul. Power, 31(2), 976-981 (2015)

[26] N. D. Sandham. "Effects of Compressibility and Shock-Wave Interactions on Turbulent Shear Flows," Flow Turbul. Combust., 97, 1-25 (2016)

[27] N. D. Sandham, E. Schülein, A. Wagner, S. Willems, and J. Steelant. "Transitional shock-wave/boundary-layer interactions in hypersonic flow," J. Fluid Mech., 752, 349-382 (2014)

[28] E. Touber. Unsteadiness in shock wave boundary layer interactions. PhD thesis, University of Southampton (2010) 
[29] E. Touber, and N. D. Sandham, Large-eddy simulation of low-frequency unsteadiness in a turbulent shock-induced separation bubble. Theor. Comput. Fluid Dyn. 23, 79-107 (2009) [30] Z. T. Xie, and I. P. Castro. "Efficient generation of inflow conditions for large-eddy simulation of street-scale flows," Flow Turbul. Combust., 81, 449-470 (2008)

[31] B. Wang, N. D. Sandham, Z. Hu, and W. D. Liu, "Numerical study of oblique shockwave/boundary-layer interaction considering sidewall effect,” J. Fluid Mech. 767, 526-561. (2015)

[32] K. W. Thompson. "Time dependent boundary conditions for hyperbolic systems,".J. Comput. Phys., 68 (1), 1-24 (1987)

[33] N. D. Sandham, Q. Li, and H. C. Yee. "Entropy splitting for high-order numerical simulation of compressible turbulence,” J. Comput. Phys., 178, 307-322 (2002)

[34] N. S. Dhamankar, G. A. Blaisdell, and A. S. Lyrintzis. "An Overview of Turbulent Inflow Boundary Conditions for Large Eddy Simulations,” AIAA 2015-3213 (2015)

[35] M. Klein, A. Sadiki, and J. Janicka. "A digital filter based generation of inflow data for spatially developing direct numerical or large eddy simulations," J. Comput. Phys., 186, 652$665(2003)$

[36] P. Schlatter, and R. Orlu. "Assessment of direct numerical simulation data of turbulent boundary layers,” J. Fluid. Mech., 659, 116-126 (2010)

[37] M. R. Gruber, A. S. Nejad, T. H. Chen, and J. C. Dutton. "Transverse Injection from Circular and Elliptic Nozzles into a Supersonic Crossflow,” J. Propul. Power, 16 (3), 449-457 (2000)

[38] A. D. Rothstein, and P. J. Wantuck. "A Study of the Normal Injection of Hydrogen Into a Heated Supersonic Flow Using Planar Laser-Induced Fluorescence,” AIAA 92-3423 (1992) 